LA W RENCE LIVERMORE N A TIONAL LABORATORY

\section{Rotational Electrophoresis of Striped Metallic Microrods}

Klint A. Rose, John A. Meier, George M.

Dougherty, Juan G. Santiago

December 1, 2005

Physical Review E 
This document was prepared as an account of work sponsored by an agency of the United States Government. Neither the United States Government nor the University of California nor any of their employees, makes any warranty, express or implied, or assumes any legal liability or responsibility for the accuracy, completeness, or usefulness of any information, apparatus, product, or process disclosed, or represents that its use would not infringe privately owned rights. Reference herein to any specific commercial product, process, or service by trade name, trademark, manufacturer, or otherwise, does not necessarily constitute or imply its endorsement, recommendation, or favoring by the United States Government or the University of California. The views and opinions of authors expressed herein do not necessarily state or reflect those of the United States Government or the University of California, and shall not be used for advertising or product endorsement purposes. 
UCRL-JRNL-217473

Rotational Electrophoresis of Striped Metallic Microrods

Klint A. Rose, ${ }^{1,2}$ John A. Meier, ${ }^{1}$ George M. Dougherty, ${ }^{2}$ and Juan G. Santiago ${ }^{1}$

${ }^{1}$ Department of Mechanical Engineering, Stanford University, Stanford, California 94305, USA

${ }^{2}$ Center for Micro and Nanotechnology,

Lawrence Livermore National Laboratory, Livermore, California 94550, USA

Analytical models are developed for the translation and rotation of metallic rods in a uniform electric field. The limits of thin and thick electric double layers are considered. These models include the effect of stripes of different metals along the length of the particle. Modeling results are compared to experimental measurements for metallic rods. Experiments demonstrate the increased alignment of particles with increasing field strength and the increase in degree of alignment of thin versus thick electric double layers. The metal rods polarize in the applied field and align parallel to its direction due to torques on the polarized charge. The torque due to polarization has a second order dependence on the electric field strength. The particles are also shown to have an additional alignment torque component due to non-uniform densities along their length. The orientation distributions of dilute suspensions of particles are also shown to agree well with results predicted by a rotational convective-diffusion equation.

\section{INTRODUCTION}

Rod-shaped metal particles with $200 \mathrm{~nm}$ to $4 \mu \mathrm{m}$ diameters and lengths of 2 to $40 \mu \mathrm{m}$ can be grown as homogenous wires or with stripes of varying materials [1,2]. In the latter case, material stripes along the particle provide a barcode that can encode on the order of 10 bits of information. In biological detection applications, the barcode is used to identify a hybridization or immunoassay reaction that can be performed and detected in parallel with reactions corresponding to many other particle types in a common chamber or microfluidic channel $[2,3]$. Homogenous nano- or microwires have been proposed as methods to construct electronic circuits, nanosensors, and biologicalelectronic interfaces [4-7]. Assembly and orientation of metal nanowires has been demonstrated using dielectrophoresis with patterned electrodes and magnetophoresis with external magnets [8-11]. Such particles can also be manipulated with uniform electric fields through electrophoretic translation and rotation $[12,13]$.

The electrophoretic motion of a particle is determined by an electrostatic force exerted on surface charge, hydrodynamic drag forces, a retarding force due to the flow of countercharges, and an electric relaxation force from the separation of positive and negative charges as the countercharges move over the particle surface [14]. Although generally these forces cannot be superimposed, their net effect on a particle moving at constant velocity (rotational or translational) must be zero. There has been extensive work on the modeling of electrophoresis of both spherical and non-spherical particles. Here we present a summary of the extensive work on modeling electrophoretic particles. 
Although these models primarily focus on non-conducting particles, we highlight aspects that are specifically relevant to ideally polarizable (i.e., non-reacting) metal particles.

The most common model for single-particle electrophoresis, developed by Smoluchowski, is derived using at least five basic assumptions [15, 16]: $\left(\lambda_{D} / a\right) \exp (z e \zeta / 2 k T)<<1$, where $k T / e$ is the thermal voltage (approximately $\left.25 \mathrm{mV}\right), \zeta$ is the zeta potential, $\lambda_{D}$ is the Debye length, and $a$ is the characteristic particle length scale; (ii) zeta potential is uniform over the surface of the particle; (iii) applied field, $E_{\infty}$, does not disturb the charge distribution in the electric double layer (EDL); (iv) the particle is rigid and dielectric (such that $\varepsilon_{p}<<\varepsilon_{m}$ where $\varepsilon_{p}$ is the particle permittivity and $\varepsilon_{m}$ is the permittivity of the liquid); and (v) the surrounding liquid is unbounded. Under these conditions,

$$
u=\frac{\varepsilon_{m} \zeta_{o}}{\eta} E_{\infty} \text { and } \omega=0,
$$

where $u$ is the translational velocity of the particle, $\omega$ is the rotational velocity of the particle, $\zeta_{o}$ is the uniform native (i.e., due to spontaneous chemical surface reactions with the electrolyte) zeta potential at the EDL shear plane, $\eta$ is the liquid viscosity, and $E_{\infty}$ is the applied field [17]. Assuming (i)-(v), Morrison [18] and Teubner [19] showed that Eq. 1 is appropriate for dielectric particles of any shape (i.e. spheres, cylinders, ellipsoids, etc).

If the EDL around a dielectric particle is thick, such that $\lambda_{D} \gg a$, the simplest model of the motion is a balance of the Coulombic force on the particle with the hydrodynamic drag. For example, a spherical particle with surface potential, $\zeta$, would have a net charge of $Q=4 \pi a \varepsilon_{m} \zeta$. The Coulombic force on the particle, $Q E_{\infty}$, is balanced by the Stokes' drag force, $6 \pi \eta a u$, to yield the Hückel equation,

$$
u=\frac{2 \varepsilon \zeta_{o}}{3 \eta} E_{\infty}
$$

for the electrophoretic velocity [20]. When $\lambda_{D}>>a$ the translational velocity of a cylinder (e.g., modeled as a prolate spheroid) is a maximum when the particle is aligned parallel to the field and a minimum when the particle is perpendicular to the field. The translational velocity at any orientation is therefore $u_{\perp} \leq u \leq u_{\|}$, where the subscripts $\perp$ and $\|$ denote a particle with the major axis oriented perpendicular and parallel to the applied field, respectively. Assuming (ii)-(v), the electrophoretic velocity of a small aspect ratio (diameter/length $<<1$ ), prolate spheroid with thick EDL is $\frac{\varepsilon \zeta_{o}}{2 \eta} E_{\infty} \leq u \leq \frac{\varepsilon \zeta_{o}}{\eta} E_{\infty}$ (where the bounds are the values of $u_{\perp}$ and $u_{\|}$) as shown by Harris [21].

The $\lambda_{D} \gg a$ analysis is useful but only a limiting behavior. For spherical particles, Henry [22] solved for an additional term, $f(\kappa a)$, that multiplies the right hand side of Eq. 2, varies from $2 / 3$ to 1 , and accounts for the retardation force caused by the motion of EDL counter ions. This term links the limiting cases described by Eq. 1 and Eq. 2. Ohshima [23] developed an expression for $f(\kappa a)$ for cylindrical particles and Yoon [24] for spheroidal particles (he uses the variable $C_{P R}$ for his multiplicative term) for the cases of the field parallel and perpendicular to the particle. Stigter developed similar results for $f(\kappa a)$ (he uses $y_{o}$ ) for highly charged cylindrical particles $[25,26]$. 
The limit of $\left(\lambda_{D} / a\right) \exp (z e \zeta / 2 k T)<<1$ in the assumptions for Eq. 1 implies that so-called surface conduction through the particle's electric double layer can be neglected. As the charge density in the double layer increases with respect to the conductivity of the bulk solution, external field lines in the vicinity of the particle bend into the double layer as though its surface were an ion conducting surface. For particles with high surface charge, $\zeta>k T / z e$, but thin EDL $\left(\lambda_{D}<<a\right)$, this high EDL charge density leads to a surface conduction tangential to the charged particle surface. The dimensionless Dukhin number, (referred to as 'Rel' in the Russian literature),

$$
D u=\frac{\sigma_{s}}{\sigma a}
$$

compares the surface conduction of the EDL, $\sigma_{s}$, to the conductivity of the bulk solution, $\sigma$, and the characteristic particle scale, $a$ (radius for spherical particles). For $D u<<1$, the field lines of applied field are unaffected by the charge in the double layer. For finite $D u$, the flow of ions through the diffuse ion layers around the particle can create charge buildup in the outer limits of the double layer at one end of the particle and depletion at the other end. This change in ion density creates concentration gradients that lead to diffusio-phoresis and a modification of the EDL which affects net electrophoretic motion [27]. O'Brien and Ward [28] derived an analytical expression for the electrophoretic translation of spheroidal particles as the zeta potential increases. Ho et al. [29] used this model to determine the zeta potential of ellipsoidal polystyrene particles with aspect ratios (diameter/length), between 1.0 and 4.5. Their observations deviated less than $10 \%$ from values obtained using Eq. 1.

The depletion of ions from some EDL regions and accumulation in others can cause significant distortion and polarization of the EDL. Charge polarization is characterized by an induced dipole moment (idm). The degree of polarization and the idm depend on applied field, particle shape, size, and orientation, and $D u$. For nonspherical particles, the external field acts on the induced dipole moment to induce a torque and rotational velocity. The induced dipole moment of dielectric rod-like particles and the resulting particle rotation has been investigated in the Russian literature [30-34] and in models of rod-like molecules [35, 36]. Dukhin and Shilov [37] reviewed much of the work on induced dipole models for spherical, ellipsoidal, and rod-like particles, and Mandel [38] reviewed induced dipole models as they apply to rod-like polyions.

In this study, we focus on (conducting) metal rod-like particles. In most cases these metal cylinders can be modeled as ideally polarizable as they are small enough such that the potential imposed by the external field along the particles is insufficient to induce electrochemical reactions. The work of Simonov and Dukhin [39] shows the translational velocity in Eq. 1 is correct for an ideally polarizable particle of any shape provided that $\left(\lambda_{D} / a\right) \exp \left(z e\left(\zeta_{o}+E_{\infty} a\right) / 2 k T\right)<<1$, where the additional term $E_{\infty} a$ is the induced potential and $a$ is the characteristic length along the direction of the electric field. Under these conditions, surface conduction through the EDL is minimal $(D u<<1)$ and diffusio-phoresis due to concentration polarization of the particle's EDL can be neglected. The particle and surrounding double layer both polarize however. Initially, electrons within the metal particle very quickly redistribute themselves through the metal to maintain a uniform electric potential at the particle surface, and field lines intersect the particle surface at right angles (FIG. 1a). For time scales shorter than $\varepsilon_{m} a / \sigma_{s} \lambda_{D}$, electrolyte ions then accumulate at the (non-reacting) particle boundaries and act to shield 
the particle from electric flux. For time scales larger than order $\varepsilon_{m} a / \sigma_{s} \lambda_{D}$, electrolyte ions completely shield electric flux from the particle and a final state is achieved where field lines are everywhere tangential to the surface (FIG. 1b). In this final state, the particle boundary acts the same as that of a perfect insulator. The time scale of the transition is limited by the electromigration of positive and negative ions from the solution to opposite sides of the particle surface, creating a screening cloud that expels field lines [40]. The charging time, $\varepsilon_{m} a / \sigma_{s} \lambda_{D}$, applies when the zeta potential does not exceed the thermal voltage $\left(\zeta_{o}+E_{\infty} a<k T / z e\right)$. As the zeta potential increases beyond the thermal voltage, the relation between double layer capacitance and charge density becomes nonlinear and the charging time becomes a function of the zeta potential (see reference [40] for a detailed discussion of charging time for systems with high zeta potential).
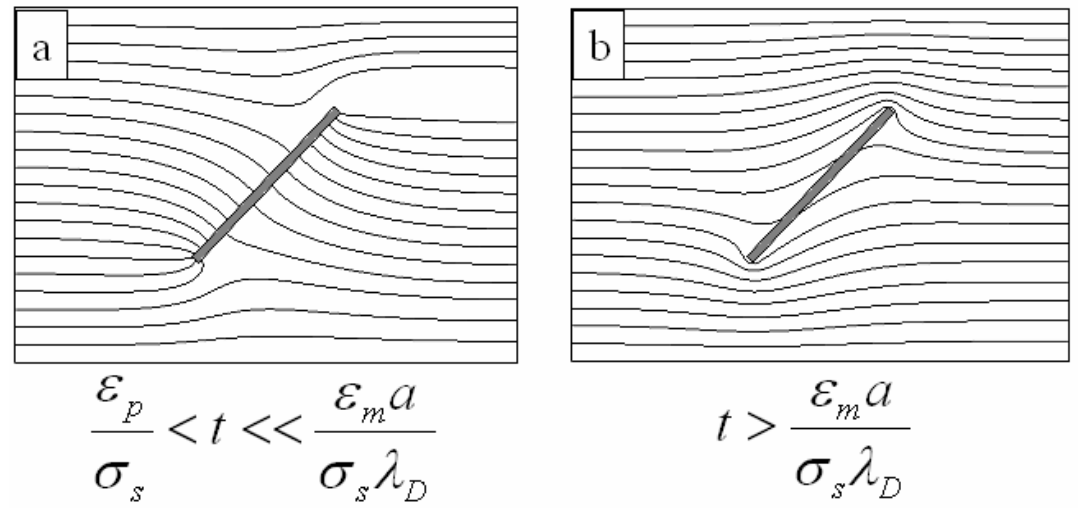

FIG. 1. Electric fields lines around an ideally polarizable rod-like particle in an electrolyte at two different times after an external field is applied. (a) Initial state nearly instantaneously after the field is applied. The relaxation time for the redistribution of electrons in the metal particle, $\tau_{R}=\varepsilon_{p} / \sigma_{s}$, is on the order of $10^{-18} \mathrm{~s}$. After this time the field lines intersect the conducting particle at right angles although no electrochemical reactions occur at the surface. (b) Final state after the electromigration of ions to the particle surface charges the double layer. This charging of the double layer occurs on a time scale of order $10^{-6} \mathrm{~s}$.

After the double layer polarizes, part of the ions within the screening cloud around the particle remain mobile and continue to migrate towards the electrode of opposite charge. These "induced" counter-charges drag fluid with them as they move through the double layer. Simonov and Dukhin [39] and Fixman and Jagannathan [41] included this effect in their analysis of the polarized charge cloud around conducting spheres and non-conducting infinite cylinders respectively. Fixman and Jagannathan [41] calculated the resulting electrophoretic velocity and dipole moment for DNA molecules. Bazant and Squires [42] and Squires and Bazant [40] described in detail the liquid flow field generated around the particle due to the motion of the induced counter-charges through the double layer. They termed this effect induced-charge electroosmosis (ICEO). For particles with broken symmetries, e.g. non-uniform surface properties, the flow due to the electromigration of positive ions may exceed that of negative ions (or vice versa) causing the particles to translate due to induced-charge electrophoresis (ICEP) [42, 43]. 
Particles with reflection symmetry, such as the rod-like particles of interest here, do not translate due to ICEP, but the motion of the "induced" counter-charges contributes to the rotation of the particle [42]. The total rotational motion of a rod-like particle in the thin EDL limit includes both the ICEP rotation and the dielectrophoretic rotation. Yariv [44] recently derived generic long time solutions for the ICEP translational and rotational velocities of arbitrary shaped conducting particles for the case of $D u<<1$. His analysis includes the dielectrophoretic contribution to translation of the particle in non-uniform electric fields and rotation of the particle in uniform or non-uniform fields. Squires and Bazant [43] extend their translational and rotational ICEP solutions to particles with broken symmetries due to partial dielectric coatings and include dielectrophoretic contributions to the motion. Saintillan et al. used slender body theory to model the induced charge electroosmotic flow at the surface of cylindrical metal particles and demonstrated increased hydrodynamic interactions of particles due to these flows [45].

For ideally polarizable rod-like particles with finite EDL, experimental measurements of zeta potentials have used variations of Eq. 2 to model translational motion $[12,13]$. This approach assumes an evenly distributed (random) distribution of particle orientations in a dilute suspension of cylinders. The average electrophoretic translation of the particles, $\langle u\rangle$, can therefore be described as

$$
\langle u\rangle=\int_{0}^{\pi / 2}\left(u_{\|} \cos \theta+u_{\perp} \sin \theta\right) \sin \theta d \theta=\frac{1}{3}\left(u_{\|}+2 u_{\perp}\right)
$$

where $\theta$ is the angle between the applied field and the major axis of the particle [46]. Applying the limiting $u_{\perp}$ and $u_{\|}$relations of Harris for $\lambda_{D}>>a$, the average electrophoretic translation of a prolate spheroid simplifies to Eq. 2. Assuming an evenly distributed assumption is accurate (as we will discuss below, this is typically not the case), the zeta potential of rod-like particles with length $>>$ diameter and thick EDLs is measurable using standard commercial zeta potential systems. Van der Zande et al. used this approach to measure the zeta potential of gold cylindrical particles with $\lambda_{D} / a$ values ranging from 1.5-6.0 [13]. Using particle tracking, Davison et al. compared experimental electrophoretic values to a modified version of Eq. 4 which included the corrections for high surface charge of Stigter $[12,25,26]$. The particles investigated were gold cylinders with $\lambda_{D} / a$ values of approximately 1.0 . The large $( \pm 30 \%)$ difference between experimental values and predictions was attributed to an orientation dependence of the particles. Both authors however, neglect translation and rotation due to the induced dipole moment of the particles. These effects can alter the measured average electrophoretic velocity of the particles. Han and Yang. have demonstrated the significance of the orientation distributions for electrophoresis of spheroidal particles, focusing specifically on models for dielectric particles [47].

In this paper, we present analytical and experimental studies of the translational and rotational electrophoresis of metal rods with either homogenous or non-homogenous surface properties for both the limiting cases of thick and thin EDL. We also explore the coupled effects of rotational electrophoresis, rotation due to gravity, and Brownian diffusion. We include the effects of induced charge electrophoresis (i.e., the effect of induced surface charges on the EDL and electrophoretic motion of the particle) and dielectrophoresis. The next section describes our models for electrophoretic particle translation and rotation and is divided into five sub-sections describing the thick EDL limit particle rotation and translation, the thin EDL limit particle rotation and translation, 
particle motion in $\mathrm{AC}$ fields, particle motion due to gravity, and a Fokker-Plank formulation for the orientation distribution of polarized particles. The Experimental Procedure section describes our experiments quantifying particle motion and orientation (including a particle image recognition and tracking code). The Results and Discussion section also shows quantitative comparisons between experiments and data. We close with Conclusions and Recommendations.

\section{THEORY}

This section describes models for particle translation, rotation, and orientation statistics. We consider limiting cases for thick EDL $\left(\lambda_{D}>>c\right)$ and thin EDL $\left(\lambda_{D}<<b\right)$ in DC fields, then extend these solutions to AC fields. We also consider settling velocities due to gravity. We model a cylindrical particle with length $L$ and radius $r$ as a prolate spheroid with characteristic half-lengths $c=L / 2$ and $b=r$, as shown in FIG. 2. Using the spheroidal geometry enables transformation to the curvilinear ellipsoidal coordinate system [48] in which the relevant Laplace's equation is separable. The axes of the coordinate system, $x_{1}^{\prime}, x_{2}^{\prime}$, and $x_{3}$, are aligned with the two minor axes (with equal lengths $b$ ) and the major axis (length $c$ ) of the particle, respectively.

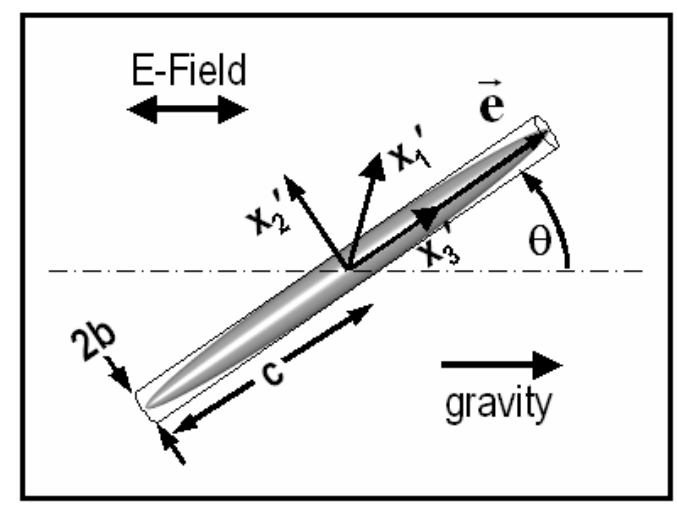

FIG. 2. Spheroid geometry used to model the cylindrical particles. The coordinate system is aligned with the $\mathrm{x}_{3}{ }^{\prime}$ component parallel to the primary axis of the particle. The directional vector, $\overrightarrow{\mathbf{e}}$, is oriented along the length of the particle and the angle of the particle is measured between this vector and the direction of the applied field. The field is applied parallel to the direction of the gravitational acceleration vector, $\overrightarrow{\mathbf{g}}$.

\section{B. Thick EDL Model}

For particles with a thick EDL, we propose an approximation for the translation and rotation of the particle due to induced charge effects using similar assumptions to those used by Hückel [20] in deriving Eq. 2. These assumptions lead us to a slight modification of dielectrophoresis results obtained by Rivette and Baygents [49] and Jones [50] for the force and torque on a rod-like metal and dielectric particles respectively. The analysis is also extended to include the effects of stripes along the length of the particle on the translation and rotation of the metal particles.

For the thick EDL case the double layer around the particle is extended and the motion of EDL counterions does not appreciably contribute to particle drag. Particle 
motion is therefore assumed to be a balance between the electrostatic force and torque on the particle and the hydrodynamic drag. This assumption was implemented by Hückel [20] to derive Eq. 2 and by Han and Yang. [47] in deriving the equations describing the translation and rotation of dielectric rod-like particles. This analysis is appropriate for spherical particles when $\lambda_{D} \gg>a_{s}$ (where $a_{s}$ is the radius of a sphere). For cylinders, however, the strictest condition for thick double layers is such that the Debye length is much larger than the half-length of the cylinder $\left(\lambda_{D}>>c\right)$. As a simple model, we here use a strict thick double layer model $\left(\lambda_{D}>>c\right)$ to estimate the physics of the intermediate double layer thickness regime when the Debye length exceeds the particle radius but is less than the particle half length $\left(b<\lambda_{D}<c\right)$. As we shall see in Section IV, the thick double layer model does very well in predicting particle alignment physics for electric double layer thicknesses larger than cylinder radius and smaller than the cylinder half length.

The thick EDL electrophoresis problem is separated into two parts: the motion due to the induced polarization of the particle (subscript DEP to denote dielectrophoresis), and the motion due to a (perhaps non-uniform) native zeta potential. Force and torque are then summed to obtain the following equations of particle motion,

$$
\begin{aligned}
& \mathbf{u}_{\text {Thick }}=\left(\frac{\mathbf{e e}}{d_{\|}}+\frac{\mathbf{I}-\mathbf{e e}}{d_{\perp}}\right) \cdot\left(\mathbf{F}_{D E P}+\mathbf{F}_{\text {nat }}\right) \text { and } \\
& \boldsymbol{\omega}_{\text {Thick }}=\left(\frac{\mathbf{e e}}{d_{\theta, \|}}+\frac{\mathbf{I}-\mathbf{e e}}{d_{\theta, \perp}}\right) \cdot\left(\mathbf{T}_{D E P}+\mathbf{T}_{\text {nat }}\right)
\end{aligned}
$$

where $d_{\|}$and $d_{\perp}$ are the translation drag coefficients and $d_{\theta, \|}$ and $d_{\theta, \perp}$ are the rotational drag for rotation about the primary and secondary axes.

The force and torque on a metal particle are due to the Maxwell stresses acting on the on the particle surface. The general expressions for this force, $\mathbf{F}_{D E P}$, and torque, $\mathbf{T}_{D E P}$, are

$$
\begin{aligned}
& \mathbf{F}_{D E P}=\int_{S_{p}} \varepsilon\left((\mathbf{n} \cdot \mathbf{E}) \mathbf{E}-\frac{E^{2}}{2} \mathbf{n}\right) d S \text { and } \\
& \mathbf{T}_{D E P}=\int_{S_{p}} \mathbf{r} \times \varepsilon\left((\mathbf{n} \cdot \mathbf{E}) \mathbf{E}-\frac{E^{2}}{2} \mathbf{n}\right) d S
\end{aligned}
$$

where $\mathbf{E}$ is the local electric field and $\mathbf{n}$ is everywhere normal to the particle surface [51, 52]. Using a multipole expansion and ignoring far-field effects, Rivette and Baygents [49] reformed Eqs. 6a-b in terms of the applied electric field, $\mathbf{E}_{\infty}$,

$$
\mathbf{F}_{D E P}=\varepsilon \int_{S_{p}}\left(\left(\mathbf{n} \cdot \mathbf{E}_{\infty}\right) \mathbf{E}_{\infty}-\frac{1}{2}\left(\mathbf{E}_{\infty} \cdot \mathbf{E}_{\infty}\right) \mathbf{n}\right) d S
$$

which simplifies to $\mathbf{F}_{D E P}=0$ since the applied field $\mathbf{E}_{\infty}$ is uniform, and

$$
\mathbf{T}_{\text {DEP }}=\left(\mathbf{M}_{p} \cdot \mathbf{E}_{\infty}\right) \times \mathbf{E}_{\infty} \text {. }
$$

The polarization tensor, $\mathbf{M}_{p}$, is derived from the dipole moment on the conducting particle under the conditions described by Eq. 17 but with the boundary condition $\phi_{\text {cond }}=0$ on the particle surface. The tensor depends on the shape of the particle and for a spheroid is as follows, 


$$
\mathbf{M}_{p}=\frac{\varepsilon V_{p}}{L_{\|}} \mathbf{e e}+\frac{\varepsilon V_{p}}{L_{\perp}}(\mathbf{I}-\mathbf{e e})
$$

Here we have expressed $\mathbf{M}_{p}$ in terms the volume of the spheroid, permittivity of the medium, and the polarization factor, $L$. The polarization factor is defined as

$$
L_{1}, L_{2}=L_{\perp}=\frac{b^{2} c}{2} \int_{0}^{\infty} \frac{d s}{\left(s+c^{2}\right)^{\frac{1}{2}}\left(s+b^{2}\right)^{2}} \text { and } L_{3}=L_{\|}=\frac{b^{2} c}{2} \int_{0}^{\infty} \frac{d s}{\left(s+c^{2}\right)^{\frac{3}{2}}\left(s+b^{2}\right)} 9
$$

which can be determined analytically in terms of the aspect ratio, $\alpha \equiv b / c(c>b)$,

$$
\begin{aligned}
& L_{\perp}=\frac{1}{2\left(1-\alpha^{2}\right)}-\frac{\alpha^{2}}{4\left(1-\alpha^{2}\right)^{3 / 2}} \ln \left(\frac{1+\left(1-\alpha^{2}\right)^{1 / 2}}{1-\left(1-\alpha^{2}\right)^{1 / 2}}\right) \\
& L_{\|}=-\frac{\alpha^{2}}{\left(1-\alpha^{2}\right)}+\frac{\alpha^{2}}{2\left(1-\alpha^{2}\right)^{3 / 2}} \ln \left(\frac{1+\left(1-\alpha^{2}\right)^{1 / 2}}{1-\left(1-\alpha^{2}\right)^{1 / 2}}\right) .
\end{aligned}
$$

The resulting dielectrophoretic translation and rotation of the spheroid particle in a uniform electric field is:

$$
\begin{aligned}
& \mathbf{u}_{D E P}=0 \text { and } \\
& \boldsymbol{\omega}_{D E P}=\left[\left(\frac{\varepsilon V_{p}}{d_{\theta,} L_{\|}} \mathbf{e e}+\frac{\varepsilon V_{p}}{d_{\theta, L} L_{\perp}}(\mathbf{I}-\mathbf{e e})\right) \cdot \mathbf{E}_{\infty}\right] \times \mathbf{E}_{\infty} .
\end{aligned}
$$

We use a similar analysis to estimate the force and torque on a striped particle due to segments of varying surface charge density, $\sigma(z)$, along the length. We can relate the surface charge to the native zeta potential through the capacitance, calculated by Jeans [53] for a conducting prolate spheroid as

$$
C_{s}=4 \pi c \varepsilon \sqrt{1-\alpha^{2}}\left(\ln \frac{1+\sqrt{1-\alpha^{2}}}{1-\sqrt{1-\alpha^{2}}}\right)^{-1} .
$$

This capacitance is for a uniform cylinder. For the case of a striped particle, we treat the contributions of the various metal stripes by linearly superposing their capacitance values (as with capacitors in parallel). We note that Eq. 13 is therefore only an approximation to the true overall capacitance value of a striped particle and should be valid when the stripe length is greater than the rod radius. For narrow stripes, this equation may not fully describe the stripe-to-stripe electrostatic interaction.

The force on the particle due to the native zeta potential is obtained by integrating over the surface area to find the net Coulombic force on the surface,

$$
\mathbf{F}_{n a t}=\frac{C_{s} \mathbf{E}_{\infty}}{2} \int_{-1}^{1} \zeta\left(Z^{\prime}\right) d Z^{\prime} .
$$

The net torque determined from the Coulombic forces and respective moment arms is

$$
\mathbf{T}_{\text {nat }}=\mathbf{p}_{1} \times \mathbf{E}_{\infty},
$$

where

$$
\mathbf{p}_{1}=\frac{C_{s} c}{2} \int_{-1}^{1} Z^{\prime} \zeta\left(Z^{\prime}\right) d Z^{\prime} \mathbf{e}
$$


The translational drag components in Eq. 5 are calculated as quasi-static (i.e., neglecting inertia) values for Stokes flow around a spheroid particle [54]. The drag on a spheroid with an aspect ratio, $\alpha<<1$, moving parallel to the primary particle axis is approximated as [55]

$$
d_{\|} \approx \frac{4 \pi \eta c}{\ln (2 / \alpha)-1 / 2}
$$

and the drag for motion perpendicular to the primary axis is approximated as

$$
d_{\perp} \approx \frac{8 \pi \eta c}{\ln (2 / \alpha)+1 / 2} .
$$

Since rotation about the primary axis is not of interest, we ignore the rotational drag component $d_{\theta, \| \cdot}$. The hydrodynamic drag coefficient for rotation about an axis perpendicular to the primary axis, $d_{\theta, \perp}$, is approximated as [56]

$$
d_{\theta, \perp} \approx \frac{8 \pi \eta c^{3}}{3[\ln (2 / \alpha)-1 / 2]} .
$$

If $\alpha<1 / 2$, the approximations in Eqs. 15 and 16 are within $1.5 \%$ of the exact solutions. Note that these drag models do not take into account electroviscous (or so-called electrolyte friction) effects on particle drag. The zeta potentials of interest here are less than or equal to the thermal voltage, and so we expect electroviscous effects to be negligible (see Stigter [57] and van de Ven [58]).

To confirm the consistency of our solution, we consider two special cases for an ideally polarizable spheroidal particle with a thick EDL and a native zeta potential that is uniform over the particle's surface. If the induced polarization is (incorrectly) neglected, the velocity in Eq. 5a for the motion of a spheroid particle oriented parallel and perpendicular to the applied field is $u_{\|}=\frac{\varepsilon \zeta_{o}}{\eta} E_{\infty}$ and $u_{\perp}=\frac{\varepsilon \zeta_{o}}{2 \eta} E_{\infty}$ respectively. Ignoring the polarization would suggest that the particle does not rotate. These translational velocities for a particle with aspect ratio $\alpha<<1$ match those suggested by Harris [21] for a dielectric particle. If the effects of induced polarization are included, the translation still reduces to the translational velocity limit proposed by Harris, although the particle will rotate. For a particle with aspect ratio $\alpha=.05$, (e.g. the particles used in this study), the values for the translational velocities are $u_{\|}=\frac{13 \varepsilon \zeta_{o}}{15 \eta} E_{\infty}$ and $u_{\perp}=\frac{17 \varepsilon \zeta_{o}}{30 \eta} E_{\infty}$.

\section{B. Thin EDL Model}

In the case of a thin EDL, double layer polarization induces a non-uniform potential along the particle surface which we refer to as the induced zeta potential. This induced zeta potential can be interpreted as the potential associated with ionic charge which is accumulated at a non-reacting particle surface. We solve for this zeta potential by subtracting the electrostatic potential at the particle surface when the field is initially applied from the potential at the surface after the double layer has polarized. The induced zeta potential is then used to find the resulting ICEP translation and rotation of homogenous metal particles. As discussed previously, particle motion in the thin EDL limit is the superposition of ICEP and dielectrophoresis (see reference [43, 59] for a 
detailed discussion of this superposition). We therefore solve for the ICEP motion and add this solution to the dielectrophoretic motion calculated from the thick EDL model. For particles with metal stripes, we consider the additional translation and rotation due to permanent dipoles generated by forces on the different surface charge for each material.

For the thin EDL case $\left(\lambda_{D}<<b\right)$, we assume the particle is suspended in a symmetric electrolyte and the potential at the particle surface, $\zeta_{o}+E_{\infty} c$, is sufficiently small to maintain the inequality $\left(\lambda_{D} / b\right) \exp \left(z e\left(\zeta_{o}+E_{\infty} c\right) / 2 k T\right)<<1$. As mentioned above, upon application of an external field, $E_{\infty}$, electrons within the metal particle quickly redistribute themselves (after a time scale of order $\varepsilon_{p} / \sigma_{p}$ ) and maintain an equipotential particle surface (as in FIG. 1a). The electrostatic potential, $\phi_{\text {cond }}$, outside the particle can be found by solving

$$
\nabla^{2} \phi_{\text {cond }}=0
$$

subject to the boundary conditions

$$
\begin{aligned}
& \phi_{\text {cond }}=\phi_{o} \text { on the particle surface and } \\
& \nabla \phi_{\text {cond }}=-E_{\infty} \text { far from the particle. }
\end{aligned}
$$

We choose the applied electric potential at the particle center to be zero, $\phi_{\infty}(\mathrm{x}=0)=0$. The potential at the surface of the particle, $\phi_{o}$, is then approximated as the "native" zeta potential of the particle, $\zeta_{o}$.

After the $\varepsilon_{m} c / \sigma_{s} \lambda_{D}$ timescale (FIG. 1b), the potential outside the particle, $\phi_{\text {ins }}$, can be found by solving,

$$
\nabla^{2} \phi_{\text {ins }}=0
$$

with the boundary conditions

$$
\begin{aligned}
& n \cdot \nabla \phi_{i n s}=0 \text { at the particle surface and } \\
& \nabla \phi_{\text {ins }}=-E_{\infty} \text { far from the particle. }
\end{aligned}
$$

The "induced" zeta potential, $\zeta_{f}$, describing the final polarized state of the double layer is obtained from the difference between the potentials of $\phi_{c o n d}$ and $\phi_{\text {ins }}$ at the potentials at the particle surface, $S_{p}$, so that $\zeta_{f}=\phi_{\text {cond }}\left(S_{p}\right)-\phi_{\text {ins }}\left(S_{p}\right)$.

The potential distribution outside a conducting spheroidal particle in a uniform field, $E_{\infty}$, is found by simplifying the known solution for an ellipsoid (in ellipsoidal coordinates) [45] to a spheroid (in spheroidal coordinates):

$$
\phi_{\text {cond }}=-E_{\infty} \cdot x^{\prime}+\sum_{i=1}^{3}\left(\zeta_{o}+E_{\infty, x_{i}} x_{i}^{\prime}\right) \frac{K_{i}(\xi)}{L_{i}}
$$

where $L_{i}$ is defined in Eq. 9. The function, $K_{i}(\xi)$, is defined as

$$
K_{1}, K_{2}=K_{\perp}=\frac{b^{2} c}{2} \int_{\xi}^{\infty} \frac{d s}{\left(s+c^{2}\right)^{\frac{1}{2}}\left(s+b^{2}\right)^{2}} \text { and } K_{3}=K_{\|}=\frac{b^{2} c}{2} \int_{\xi}^{\infty} \frac{d s}{\left(s+c^{2}\right)^{\frac{3}{2}}\left(s+b^{2}\right)} 20
$$

where the integration bound, $\xi$, is the spheroidal coordinate describing the spheroid surfaces which are confocal with the particle surface. $\mathrm{s}$ is a dummy integration variable. The solution for the potential $\phi_{\text {cond }}\left(S_{p}\right)$ is specified in the boundary conditions for Eq. 17 as $\zeta_{o}$ and is the value obtained when integrating Eq. 19 over the surface of the particle $\left(\xi_{s}=0\right)$. 
Next, the solution for the potential distribution outside an insulating spheroidal particle in a uniform field, $E_{\infty}$, is

$$
\phi_{\text {ins }}=-E_{\infty} \cdot x^{\prime}+\sum_{i=1}^{3}\left(\zeta_{o}+E_{\infty, x_{i}} x_{i}{ }^{\prime}\right) \frac{K_{i}}{\left(1-L_{i}\right)}
$$

Again, the solution is found by simplifying the known solution for an ellipsoid (in ellipsoidal coordinates) [45] to a spheroid (in spheroidal coordinates) [51]. The potential at the surface of the insulating spheroid, $\phi_{\text {ins }}\left(S_{p}\right)$, is then

$$
\phi_{\text {ins }}\left(S_{p}\right)=-\frac{E_{\infty, x_{1}} x_{1}{ }^{\prime}}{\left(1-L_{\perp}\right)}-\frac{E_{\infty, x_{2}}{ }^{\prime} x_{2}{ }^{\prime}}{\left(1-L_{\perp}\right)}-\frac{E_{\infty, x_{3}}{ }^{\prime} x_{3}{ }^{\prime}}{\left(1-L_{\|}\right)},
$$

and the resulting induced zeta potential is

$$
\zeta_{f}\left(S_{p}\right)=\zeta_{o}+\frac{E_{\infty, x_{1}} x_{1}{ }^{\prime}}{\left(1-L_{\perp}\right)}+\frac{E_{\infty, x_{2}}{ }^{\prime} x_{2}{ }^{\prime}}{\left(1-L_{\perp}\right)}+\frac{E_{\infty, x_{3}} x_{3}{ }^{\prime}}{\left(1-L_{\|}\right)} .
$$

Next, we use the sum of the native and induced zeta potentials as a boundary condition to a continuum flow balance of viscous and electric forces around the particle. The electrophoretic particle motion is found by solving the Stokes equations with an additional term to account for the body force on the charge of the EDL resulting from $\zeta_{f}$. We separate this boundary problem into two regions, the "inner" and "outer" regions, and solve using a method of matched asymptotic expansions. The inner region extends to the edge of the double layer, $S_{p}{ }^{+}$, where the body force acting on the charge, $\rho_{e} E$, drives the flow. In the outer region, the liquid outside the double layer, the charge density, $\rho_{e}$, is essentially zero and the Stokes equation reduces to its classical form. The velocity at $S_{p}{ }^{+}$, determined from this matched asymptotics analysis, produces a net rotation and translation of the particle formulated using the Lorentz reciprocal theorem. Fair and Anderson [60] implemented this technique for an ellipsoidal particle to obtain the following solutions for the translation and rotational velocities of an ellipsoidal particle subject to an arbitrary slip velocity field, $\mathbf{v}_{s}$,

$$
\begin{aligned}
& \mathbf{u}=-\frac{1}{3 V_{p}} \iint_{S_{p}^{+}}(\mathbf{n} \cdot \mathbf{r}) \mathbf{v}_{s} d S \text { and } \\
& \mathbf{\omega}=-\frac{1}{V_{p}} \mathbf{G} \cdot \iint_{S_{p}^{+}}(\mathbf{n} \cdot \mathbf{r}) \mathbf{r} \times \mathbf{v}_{s} d S
\end{aligned}
$$

where $\mathbf{u}$ is the translation velocity of the center of mass and $\omega$ is the rotational velocity vector. For a spheroid, $\mathbf{G}=\left(2 b^{2}\right)^{-1} \mathbf{e e}+\left(b^{2}+c^{2}\right)^{-1}(\mathbf{I}-\mathbf{e e})$, and $V_{p}=4 / 3 b^{2} c$ is the volume of the particle. $\mathbf{e}$ is a unit vector along the primary axis. For electrophoretic motion, the slip velocity at the shear plane around the particle is

$$
\mathbf{v}_{s}=-\frac{\varepsilon \zeta}{\eta} \mathbf{E}_{s}
$$

which is equivalent in magnitude to Eq. 1 . In the current problem, $\zeta$ may vary over the particle's surface and the vector, $\mathbf{E}_{\mathrm{s}}$, is the "local" electric field at $S_{p}{ }^{+}$. The local field is determined from the gradient of Eq. 22, 


$$
\mathbf{E}_{s}=-\nabla \phi_{i n s}=(\mathbf{I}-\mathbf{n n}) \cdot\left(\frac{\mathbf{e e}}{\left(1-L_{\|}\right)}+\frac{(\mathbf{I}-\mathbf{e e})}{\left(1-L_{\perp}\right)}\right) \cdot \mathbf{E}_{\infty} .
$$

Together, Eqs. 23-26 provide the ICEP translational and rotational velocities of an ideally polarized spheroidal metal particle with any arbitrary native zeta potential, $\zeta_{0}$. Since the zeta potential is a scalar value, $\mathbf{u}_{\text {Thin }}$ and $\boldsymbol{\omega}_{\text {Thin }}$ can be represented as

$$
\begin{array}{lll}
\mathbf{u}_{\text {Thin }}=\mathbf{u}_{D E P}+\mathbf{u}_{I C E P}+\mathbf{u}_{\text {nat }} \text { and } & 27 \mathrm{a} \\
\boldsymbol{\omega}_{\text {Thin }}=\boldsymbol{\omega}_{D E P}+\boldsymbol{\omega}_{I C E P}+\boldsymbol{\omega}_{\text {nat }} & 27 \mathrm{~b}
\end{array}
$$

where $\mathbf{u}_{D E P}$ and $\boldsymbol{\omega}_{D E P}$ are the translation and rotation due to dielectrophoresis defined in Eq. 12, $\mathbf{u}_{I C E P}$ and $\omega_{I C E P}$ are due to ICEP, and $\mathbf{u}_{\text {nat }}$ and $\boldsymbol{\omega}_{\text {nat }}$ are due to the native zeta potential. The translation due only to ICEP is

$$
\mathbf{u}_{I C E P}=-\frac{\varepsilon}{3 V_{p} \eta} \iint_{S_{p}^{+}}(\mathbf{n} \cdot \mathbf{r}) \mathbf{E}_{s} \phi_{i n s} d S
$$

which is identically zero. The rotation due to ICEP is

$$
\boldsymbol{\omega}_{I C E P}=-\frac{\varepsilon}{V_{p} \eta} \mathbf{G} \cdot \iint_{S_{p}^{+}}(\mathbf{n} \cdot \mathbf{r}) \mathbf{r} \times \mathbf{E}_{s} \phi_{i n s} d S .
$$

These results demonstrate that the polarization causes particle rotation but does not affect translation, as polarization does not change the net charge of the particle/EDL system. This holds for metal particles with and without uniform zeta potentials.

The native zeta potential of striped-metal particles will vary along the length of the particle due to the varying surface chemistry. The zeta potential is therefore only a function of the location along the length, $\zeta_{o}=\zeta_{o}\left(z^{\prime}\right)$. In this case, the translation and rotation of the particle are [60]

$$
\begin{aligned}
& \mathbf{u}_{n a t}=\frac{\varepsilon}{\eta}\left(\frac{a_{o}}{2} \mathbf{I}-\frac{\mathbf{A}_{2, \|} \mathbf{e}+\mathbf{A}_{2, \perp}(\mathbf{I}-\mathbf{e e})}{2}\right) \cdot \mathbf{E}_{\infty} \text { and } \\
& \boldsymbol{\omega}_{n a t}=\frac{3 \varepsilon}{4 \eta c} \mathbf{a}_{1} \times \mathbf{E}_{\infty} .
\end{aligned}
$$

The terms $\mathrm{a}_{0}, \mathbf{a}_{1}$, and $\mathbf{A}_{2}$ are determined from the distribution $\zeta_{\mathrm{o}}\left(\mathrm{Z}^{\prime}\right)$ with $\mathrm{Z}^{\prime}=\mathrm{Z}^{\prime} / \mathrm{c}$ :

$$
\begin{aligned}
& a_{o}=\int_{-1}^{1} \zeta_{o}\left(Z^{\prime}\right) d Z^{\prime}, \\
& \mathbf{a}_{1}=\int_{-1}^{1} \frac{Z^{\prime} \zeta_{o}\left(Z^{\prime}\right)}{\left(1-L_{\perp}\right)}\left(\frac{1+\alpha^{2}+Z^{\prime 2}\left(\alpha^{2}-1\right)}{1+\alpha^{2}+Z^{\prime 2}\left(\alpha^{4}-1\right)}\right) d Z^{\prime} \mathbf{e}, \\
& \mathbf{A}_{2, \|}=\int_{-1}^{1} \zeta_{o}\left(Z^{\prime}\right)\left(1+\frac{Z^{\prime 2}-1}{\left(1-L_{\|}\right)\left(1+\left(\alpha^{2}-1\right) Z^{\prime 2}\right)}\right) d Z^{\prime}, \text { and } \\
& \mathbf{A}_{2, \perp}=\int_{-1}^{1} \zeta_{o}\left(Z^{\prime}\right)\left(1-\frac{1+\left(2 \alpha^{2}-1\right) Z^{\prime 2}}{2\left(1-L_{\perp}\right)\left(1+\left(\alpha^{2}-1\right) Z^{\prime 2}\right)}\right) d Z^{\prime} .
\end{aligned}
$$

Two interesting cases arise for an ideally polarizable spheroidal particle with a native zeta potential that is uniform over the particle's surface. If the induced 
polarization is (incorrectly) ignored, the velocity in Eq. 29 reduces to Eq. 1 and rotation is zero, consistent with the results of Morrison [18] and Teubner [19]. If the effects of induced polarization are included, the translation still reduces to Eq. 1, consistent with the results of Simonov and Dukhin [39], but the particle rotates.

Eq. 29b shows that applied fields can generate rotational velocities on particles with non-uniform native zeta potentials. In such cases, particle orientation is a function of the zeta potential distribution along the particle. In most cases, the particle will align with the more positively charged end towards the low potential electrode and the more negatively charged end towards the high potential electrode. The rotations described by Eqs $12 \mathrm{~b}$ and $28 \mathrm{~b}$ also tend to align the particles with the applied field. Since the polarization is dependent on orientation, this rotational displacement will be less than ninety degrees (assuming no Brownian motion). The rotational velocities in Eq. 27b superpose linearly and generally compliment one another. Although note that the case can arise in which $\omega_{\text {nat }}$ may oppose $\omega_{D E P}$ and $\omega_{I C E P}$ if, for example, the particle were initially oriented with the more positively charged end towards the high potential electrode.

\section{AC Field Effect}

The results in Sections A and B for direct current (DC) applied fields can be modified for alternating current (AC) fields. This modification is often necessary since $\mathrm{AC}$ fields are commonly used in electrophoresis experiments to avoid electroosmosis of the bulk fluid. The applied field may be written as $\mathbf{E}_{\infty}\left(\mathbf{x}^{\prime}, t\right)=\mathbf{E}_{\infty}\left(\mathbf{x}^{\prime}\right) \operatorname{Re}\left[\exp \left(i \omega_{0} t\right)\right]$, where $\mathbf{E}_{\infty}\left(\mathbf{x}^{\prime}\right)$ is the spatial component of the applied field, $i=\sqrt{-1}, \omega_{o}$ is the angular frequency of the applied field, $t$ is time, and Re denotes the real part of the expression. The time-averaged translation and rotation may be computed by substituting the field $\mathbf{E}_{\infty}\left(\mathbf{x}^{\prime}, t\right)$ for $\mathbf{E}_{\infty}\left(\mathbf{x}^{\prime}\right)$ in the velocities in Eqs. 5 and 27 then integrating them as [49]

$$
\begin{aligned}
& \langle\mathbf{u}\rangle=\frac{\omega_{o}}{2 \pi} \int_{0}^{2 \pi / \omega_{o}} \mathbf{u}\left(x^{\prime}, t\right) d t \text { and } \\
& \langle\boldsymbol{\omega}\rangle=\frac{\omega_{o}}{2 \pi} \int_{0}^{2 \pi / \omega_{o}} \boldsymbol{\omega}\left(x^{\prime}, t\right) d t .
\end{aligned}
$$

The resulting time-averaged velocities in a spatially uniform field will be $\langle\mathbf{u}\rangle=0$ for all translational velocities. Similarly, the time-averaged rotational velocities due to the permanent dipole on a particle with thick or thin EDL will be $\left\langle\boldsymbol{\omega}_{\text {nat }}\right\rangle=0$. Time averaging the rotational velocities due to the induced polarization reduces the velocities by a factor of $1 / 2$ such that $\left\langle\omega_{I C E P}\right\rangle=\omega_{I C E P} / 2$ and $\left\langle\omega_{D E P}\right\rangle=\omega_{D E P} / 2$. The polarizability of a dielectric particle in an AC field becomes complex with a dependence on complex permittivity of the particle and the medium. For metal particles, the permittivity term in the velocity equations remains real.

\section{Gravitational Settling}

Gravitational forces also contribute to the translation and rotation of rod-like metal particles. The settling problem is separated into two parts: the motion due to gravity and the motion due to buoyancy. Force and torque are summed to obtain the following equations for particle motion, 


$$
\begin{aligned}
& \mathbf{u}_{s}=\left(\frac{\mathbf{e}}{d_{\|}}+\frac{\mathbf{I}-\mathbf{e e}}{d_{\perp}}\right) \cdot\left(\mathbf{F}_{\text {grav }}-\mathbf{F}_{\text {buoy }}\right) \text { and } \\
& \boldsymbol{\omega}_{s}=\left(\frac{\mathbf{e e}}{d_{\theta, \|}}+\frac{\mathbf{I}-\mathbf{e e}}{d_{\theta, \perp}}\right) \cdot\left(\mathbf{T}_{\text {grav }}-\mathbf{T}_{\text {buoy }}\right)
\end{aligned}
$$

with drag components, $d$, from Eqs. 15 and 16 [55]. The gravitational and buoyant forces on a spheroidal particle with non-uniform density along its length, $\rho_{p}\left(Z^{\prime}\right)$, are defined as

$$
\begin{aligned}
& \mathbf{F}_{\text {grav }}=m_{p} \mathbf{g}=\pi a^{2} c \int_{-1}^{1} \rho_{p}\left(Z^{\prime}\right)\left(1-Z^{\prime 2}\right) d Z^{\prime} \mathbf{g} \text { and } \\
& \mathbf{F}_{\text {buoy }}=m_{f} \mathbf{g}=V_{p} \rho_{f} \mathbf{g}
\end{aligned}
$$

where $m_{p}$ is the mass of the particle, $m_{f}$ and $\rho_{f}$ are the mass and density of the fluid displaced by the particle, and $\mathbf{g}$ is the gravitational acceleration vector shown in FIG. 2. For a uniformly dense particle, the density function can be replaced by a constant value, $\rho_{p}$, and the gravity force reduces to $\mathbf{F}_{g r a v}=V_{p} \rho_{p} \mathbf{g}$.

The settling velocity resulting from the forces in Eq. 33 depends on the particle orientation which may be affected by gravitational and buoyant torques. The gravitational torque on a spheroidal particle with non-uniform density along its length is

$$
\mathbf{T}_{\text {grav }}=m_{p} \mathbf{r}_{c m} \times \mathbf{g}=\pi a^{2} c^{2} \int_{-1}^{1} \rho_{p}\left(Z^{\prime}\right)\left(1-Z^{\prime 2}\right)\left(Z^{\prime}-Z_{c m}^{\prime}\right) d Z^{\prime} \mathbf{e} \times \mathbf{g},
$$

with the moment arm about the center of mass, $\mathbf{r}_{\mathrm{cm}}$, defined as

$$
\mathbf{r}_{c m}=Z^{\prime}-Z_{c m}^{\prime}=Z^{\prime}-\frac{\pi a^{2} c}{m_{p}} \int_{-1}^{1} \rho_{p}\left(Z^{\prime}\right)\left(1-Z^{\prime 2}\right)\left(Z^{\prime}\right) d Z^{\prime} .
$$

The buoyant torque on a spheroidal particle with moment arm about the center of buoyancy, $\mathbf{r}_{\mathrm{cm}}$, is

$$
\mathbf{T}_{\text {buoy }}=m_{f} \mathbf{r}_{c b} \times \mathbf{g}
$$

which simplifies to $\mathbf{T}_{\text {buoy }}=0$ for spheroidal particles. Particles can have a significant gravitational rotation due to the large difference in specific gravity between the contrasting metals in the particle stripes. The resulting velocities from the gravitational force may be superimposed with the electrophoretic solutions for DC or AC fields to determine the net motion of the particle.

\section{E. Fokker-Planck Formulation for Orientation Distribution}

The rotational velocities described by Eqs. 5 and 27 above for the respective limits of $\lambda_{D} \gg>c$ and $\lambda_{D}<<b$ tend to align particles toward a preferred orientation with respect to the applied field. For the case of a spatially uniform field, particles are aligned with the major axis parallel to the applied field. If the field is applied parallel to the direction of gravity, particle alignment may be enhanced by a gravitational torque. This (deterministic) alignment due to rotational electrophoresis and gravity is counterbalanced by the (entropic) forces of diffusion. A population of particles (in a dilute mixture of particles with large particle-to-particle distances) will then acquire a probability density function (PDF) describing the orientation distribution, $\psi$. This distribution is governed by the Fokker-Planck equation 


$$
\frac{\partial \psi}{\partial t}+\nabla \cdot\left(\psi \boldsymbol{\omega}-\mathbf{D}_{\theta} \cdot \nabla \psi\right)=0
$$

in which $\boldsymbol{\omega}$ is the angular velocity vector $\left(\langle\omega\rangle\right.$ in an $\mathrm{AC}$ field) and $\mathbf{D}_{\theta}$ is the coefficient tensor of rotational diffusion [61]. This equation describes the rotational convective diffusion of the particles assuming negligible particle-particle interactions. For an axisymmetric particle such as a spheroid, $\omega$ is the angular velocity about the particle's minor axis and $\mathbf{D}_{\theta}$ becomes a scalar quantity,

$$
D_{\theta}=k T / d_{\theta, \perp}=\frac{3 k T}{8 \pi \eta c^{3}}[\ln (2 c / b)-1 / 2]
$$

describing the rotational diffusion coefficient perpendicular to the primary axis with $d_{\theta, \perp}$ defined by Eq. 16. Assuming the distribution has reached a steady-state and rotation into the plane (angle $\varphi$ ) is ignored, Eq. 36 can be non-dimensionalized as

$$
\frac{d}{d \theta}\left[\left(P e_{p o l} \sin \theta \cos \theta+P e_{p e r m} \sin \theta\right) \sin \theta+\sin \theta \frac{d \psi}{d \theta}\right]=0
$$

where $P e_{p o l}=\left\|\omega_{\text {pol }}\right\| / D_{\theta}$ and $P e_{\text {perm }}=\left\|\omega_{\text {perm }}\right\| / D_{\theta}$ are the rotational Peclet numbers for the induced (DEP and ICEP) and permanent dipoles, respectively. The permanent dipole parameter may include rotation due to gravity or non-uniform native zeta potentials in a DC field. The solution of $\psi(\theta)$ must satisfy the integral normalization condition,

$$
2 \pi \int_{0}^{\pi} \psi(\theta) \sin \theta \mathrm{d} \theta=1 .
$$

This general equation applies to either the thin or thick EDL case since we use a nondimensional $P e$. Analytical and numerical solutions to Eq. 38 were developed to ensure consistency. The analytical solution is obtained by direct integration of Eq. 38:

$$
\psi(\theta)=\psi_{o} \exp \left(\frac{P e_{p o l}}{2}\left(\cos ^{2} \theta-1\right)+P e_{p e r m} \cos \theta\right) .
$$

The normalization value of the PDF, $1 / \psi_{o}$, is difficult to obtain analytically and is here calculated numerically.

We have confirmed our solution for $\psi(\theta)$ given by Eq. 40 using a Monte Carlo simulation of the rotational electrophoresis and diffusion problem. The solution of the rotational Langevin equation [62] is

$$
\frac{I}{d_{\theta, \perp}} \frac{d \omega_{L}(t)}{d t}+\omega_{L}(t)=\omega(t)+\frac{\lambda(t)}{d_{\theta, \perp}}
$$

in which the dynamics of the particle are modeled for individual time steps to determine the overall orientation statistics $[61,63]$. In Eq. $41, I$ is the moment of inertia of the particle, $\omega_{L}(t)$ is the angular velocity of the particle at time $t, \omega(t)$ is the velocity due to the external field (Eq. 5b for thick EDL or Eq. 27b for thin EDL plus Eq. 32b for gravity), and $\lambda(t) / d_{\theta, \perp}$ is the normalized white noise driving torque due to Brownian motion. The inertial term is ignored as the particle dimensions are on the order of a micron. A comparison of the normalized analytical result in Eq. 40 and numerical results (from Monte Carlo simulations of Eq. 41) is shown in FIG. 3 for varied combinations of $P e_{p o l}$ and $P e_{p e r m}$. The Monte Carlo solution used 1000 time steps and 10,000 non-interacting particles for each combination of $P e_{p o l}$ and $P e_{p e r m}$ values. 


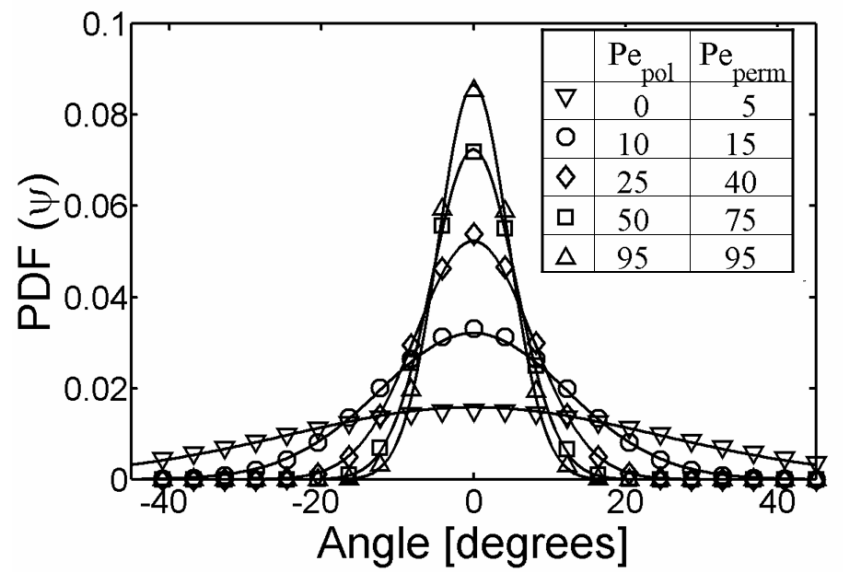

FIG. 3. Comparison of analytical and numerical solutions for the normalized orientation distributions of spheroidal particles with a dipole contribution from polarization and a permanent dipole from native surface charge and/or gravity. Several Peclet number combinations were selected to confirm consistency between the solutions over a range of conditions. The symbols show selected data points for the orientation distribution solution solved numerically using a Monte Carlo solution of the Langevin equation. The solid lines are the analytical solutions to the Fokker-Planck equation for the orientation distribution.

The Fokker-Planck analysis and the Monte Carlo simulation methods are consistent, and the normalization method for the analytical solution robust. For even moderate values of either $P e_{\text {nat }}$ or $P e_{\text {perm }}$, the orientation distribution is significantly nonuniform. This shows that assumptions of equally-distributed orientation for the purpose of estimating translation motion may be incorrect even at relatively low fields, especially for particles with a non-uniform native zeta potential. Eq. 4 in fact requires a modification to weight the translational velocities by the shape of $\psi(\theta)$ :

$$
\langle u\rangle=\int_{0}^{\pi / 2} \psi(\theta)\left(u_{\|} \cos \theta+u_{\perp} \sin \theta\right) \sin \theta d \theta .
$$

Eq. 42 describes the average (over a population of dilute particles) translational velocity as determined by the rotational orientation distribution.

\section{EXPERIMENTAL PROCEDURE}

We have performed experiments quantifying the motion and rotational distribution of metal rod-like particles subject to electric fields. We use a dilute particle solution and both relatively high and low conductivity solutions to explore the effects of double layer thickness. The particles were aligned in an AC electric field to minimize net translation due to electrophoresis. We use a setup where particles are allowed to settle along the axis of a channel, and use custom particle image recognition and tracking routines to quantify particle motion. We use this data to validate our particle rotational electrophoresis models. 


\section{A. Particle Imaging Setup}

The particle orientation experiments were performed in a borosilicate capillary with a $200 \mu \mathrm{m}$ by $2 \mathrm{~mm}$ rectangular cross section (Vitrocom, Mountain Lakes, NJ). The capillary was $10 \mathrm{~cm}$ in length and mounted vertically using an acrylic fixture to minimize interaction of particles with capillary walls as they settle. A schematic of the experimental setup is shown in FIG. 4.

The particles investigated were pure silver (111111), pure gold (000000), and fifty percent gold and silver (111000) Nanobarcode ${ }^{\circledR}$ particles (Nanoplex, Menlo Park, $\mathrm{CA})$. The binary code identifies the particle type where 1 indicates silver and 0 gold. They have lengths of $6 \mu \mathrm{m}+/-30 \mathrm{~nm}$ and diameters of $318 \mathrm{~nm}+/-50 \mathrm{~nm}[1,3]$. The two working liquids were deionized water $(\mathrm{pH} 5.5,2 \mu \mathrm{S} / \mathrm{cm}$ ) and potassium chloride solution

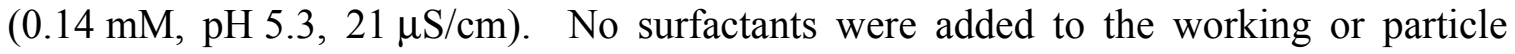
solutions. For each experiment, a single particle type was introduced into the capillary along with a working solution and allowed to equilibrate to eliminate any flow due to a pressure head. The particles were electrokinetically oriented by an alternating electric field applied along the length of the capillary with platinum wires. The alternating field eliminated any net electroosmotic or pressure driven flows in the capillary. A Trek 10/10B amplifier amplified the field supplied from an Agilent 3200 waveform generator.

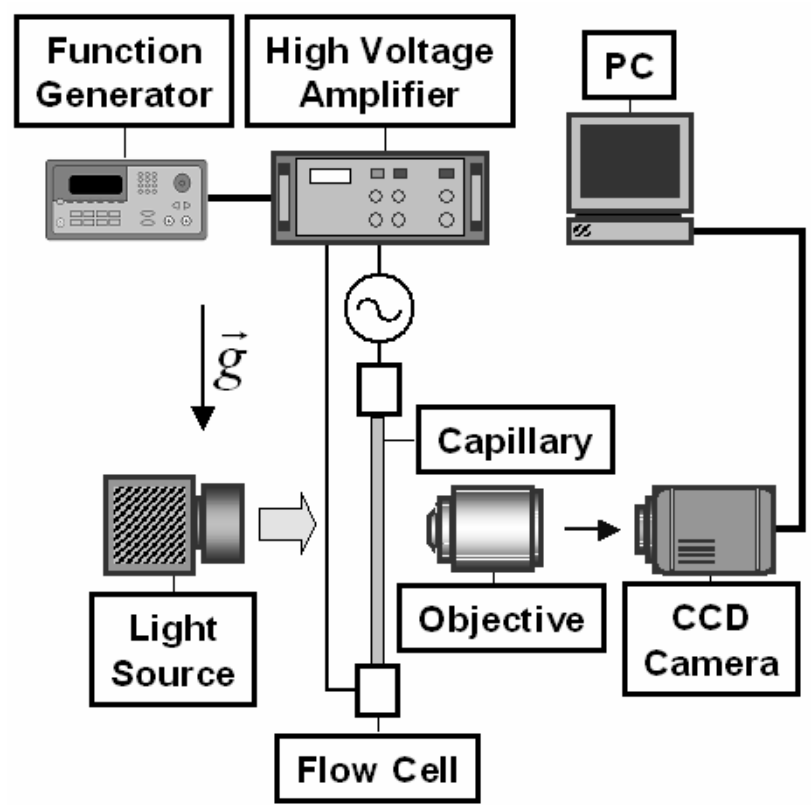

FIG. 4. Schematic of the experimental setup for particle imaging experiments. Particles flow through a capillary supported by a flow cell oriented perpendicular to the gravitational vector. The ends of the flow cell are connected to a high voltage amplifier that supplies the amplified AC signal from a function generator. The particles are imaged using a CCD camera and 60x magnification objective. Particle illumination is provided using forward scatter from a halogen light source. 
The imaging system, shown in FIG. 4, consists of a Nikon inverted epifluorescent microscope with a ninety-degree mirror placed between the objective turret and the objective; this changes the vertical object plane to a horizontal image plane. Back illumination through the channel was provided by an external halogen light source with a fiber optic light guide (Carl Zeiss MicroImaging). A Nikon oil immersion objective $(\mathrm{M}=60$, NA 1.4) with a working distance of $210 \mu \mathrm{m}$ was used to view the center plane of the capillary. Images of the particles within the capillary were recorded using a Cooke Pixelfly CCD camera (Cooke Corporation, Romulus, MI) with a 640 by 480 pixel array and 12-bit readout resolution. The camera was externally triggered with a pulse generator (Berkeley Nucleonics Corporation, San Rafael, CA) to obtain images at frame rates ranging from 1 to $5 \mathrm{fps}$. A 0.6x demagnifying lens was included in the optical path on the camera port to enable the CCD array to capture a larger field of view with a negligible loss in image resolution. A total of 1061 images were taken for each experiment. FIG. 5 shows representative images of particles settling through the flow cell with and without an applied field. Each of the images in FIG. 5 were obtained by arithmetically summing nine images with low particle density.

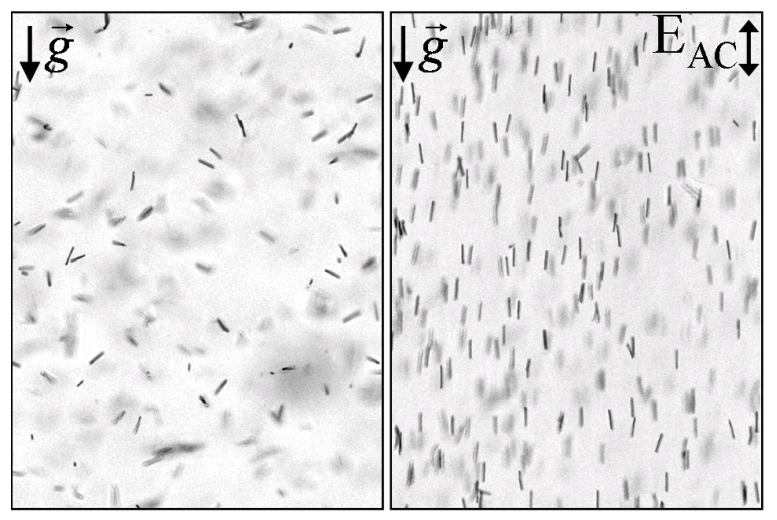

(a)

(b)

FIG. 5. Pure silver particles in DI water imaged as they settle through the flow cell. (a) Summation of 9 images at $3.3 \mathrm{~s}$ intervals showing the particles settle in random orientations without an applied field. Typical settling velocities are on the order of a few microns per second and vary depending on orientation. (b) Summation of 9 images at $3.3 \mathrm{~s}$ intervals showing particles in an AC field of $100 \mathrm{~V} / \mathrm{cm}$ at $100 \mathrm{~Hz}$ applied along the gravity vector. The field aligns the particles as they settle causing them to settle faster than randomly oriented particles.

Typically, we limit particle density so that we obtain 5 to 10 particles in each image (corresponding to particle number densities of $7.7 \mathrm{e}^{4}$ to $1.5 \mathrm{e}^{5}$ particles $/ \mu \mathrm{L}$ and $\mathrm{nL}^{3}$ values of $2 \mathrm{e}^{-3}$ to $\left.4 \mathrm{e}^{-3}\right)$. Three example two-dimensional probability density functions of particle locations are shown in FIG. 6. The figure shows the probability density of finding some part of the particle surface at some position relative to the particle center. Data from three representative field strengths are shown.

The effects of image noise in the experimental data required that we perform measurements of the particle rotational diffusivity. We measured this value for $\mathrm{Ag}$ 
particles at ten frame rates varying from 2 to $20 \mathrm{fps}$. The particles were imaged while settling through the vertical capillary with no applied electric field.
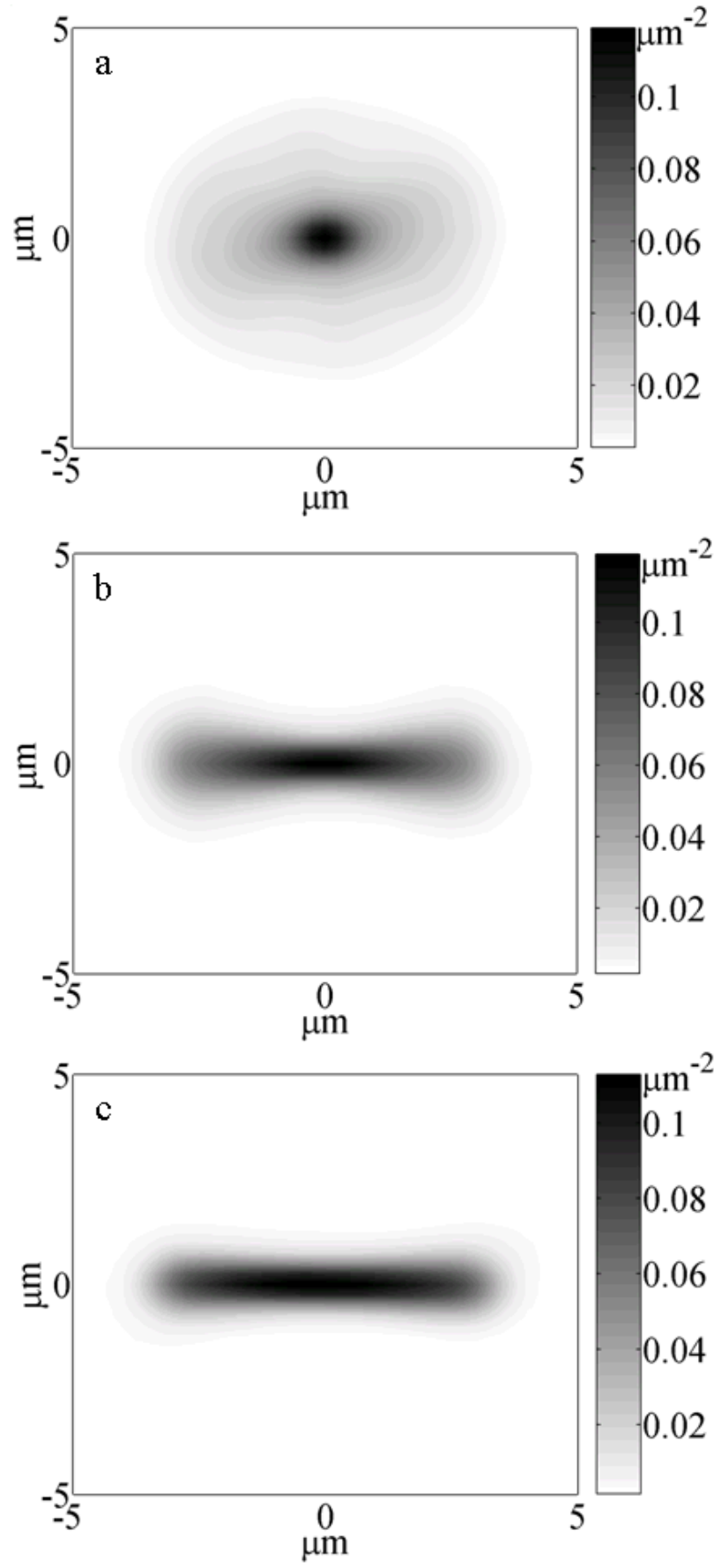

FIG. 6. Measurements of two dimensional probability density functions of $\mathrm{Ag}$ particles in deionized water determined from image data. Each PDF demonstrates the probability density of finding some part of the particle at each given location relative to its center. PDFs were constructed by analyzing images of approximately 5000 particles at three electric fields. (a) PDF for an applied field of $10 \mathrm{~V} / \mathrm{cm}$. At this field strength, the torque on the particles due to polarization is relatively weak compared to their thermal energy and particles are only weakly aligned. (b) PDF for an applied field of $50 \mathrm{~V} / \mathrm{cm}$. The electric torque more strongly aligns particles in the direction of applied field although the effect of diffusion is still apparent. (c) PDF for an applied field of $90 \mathrm{~V} / \mathrm{cm}$. At this field strength, the rotational electrophoresis dominates over the effects of diffusion.

We also performed experiments with a $30 \mathrm{~V} / \mathrm{cm}$ sinusoidal field and applied frequencies ranging from 100 to $800 \mathrm{~Hz}$ using pure silver particles in potassium chloride

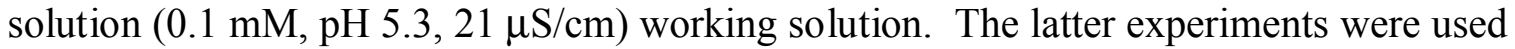
to select an alternating field frequency that ensured quasi-steady particle motion while 
exceeding the detectable limit of discrete oscillations in the translation of the metallic particles. Discrete oscillations were undetectable in frequencies above $10 \mathrm{~Hz}(100 \mathrm{~ms}$ period) for the conditions explored. The $10 \mathrm{~ms}$ period (or $100 \mathrm{~Hz}$ frequency) of the alternating field was beyond all relevant timescales for the particle motion. This is confirmed in FIG. 7 which shows a plot of measured rotational Peclet numbers obtained from imaged orientation distributions versus the period of the applied field. The rotational Peclet numbers are constant in the low frequency region of interest as the particle motion is in the electrostatic charging regime.

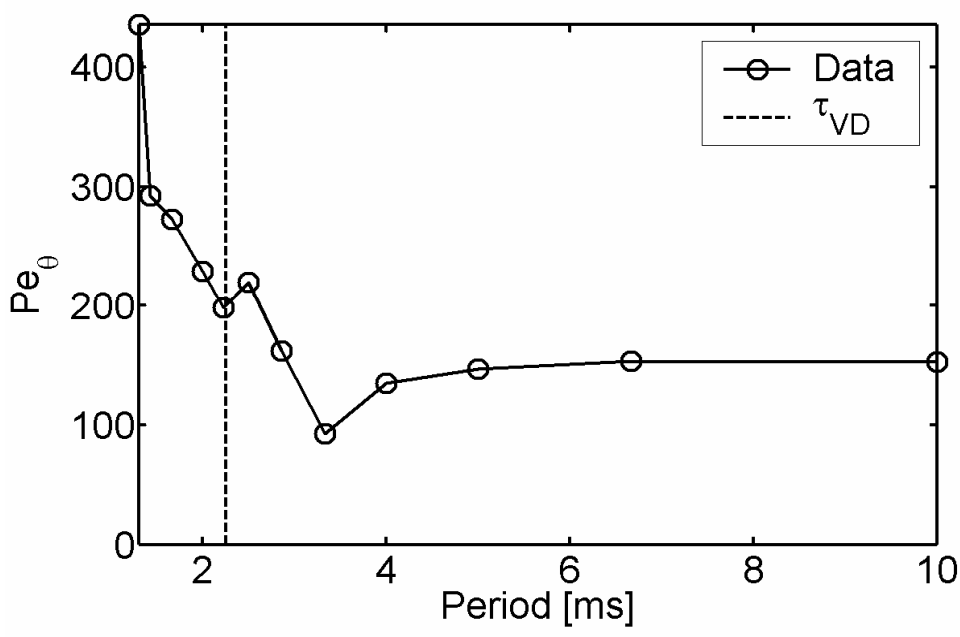

FIG. 7. Peclet number of experimentally determined orientation distributions of homogenous Ag particle for an applied field of $30 \mathrm{~V} / \mathrm{cm}$. The period of the field is varied from $1.25-10 \mathrm{~ms}$ to show the quasi-steady nature of the particle alignment beyond time scale $\tau_{V D}=a^{2} / D_{e f f}$, for diffusion through the double layer.

\section{B. Particle Tracking Velocimetry}

Particle images were analyzed using a custom particle tracking code written in MATLAB (The MathWorks, Inc., Natick, MA). Each image was interrogated to find the major axis angle and the coordinates of the particle image centroid. The code analyzes the projection of the particle orientation onto the image plane (the depth of field was $2.8 \mu \mathrm{m})$. Particle positions in succeeding image pairs were then compared to determine particle translation and rotational motion. Individual particles were identified across images using a "best match" routine described below.

A typical experiment consisted of a series of 1061 images of particles. Particles settled at approximately $4 \mu \mathrm{m} / \mathrm{s}$ and so each particle was imaged about 90 times before leaving the field of view. The average image from each experiment was subtracted from individual images to remove intensity bias from non-uniform illumination. The images were then filtered using a Weiner filter to reduce white noise, and then binarized based on pixel intensity. Particles were located in the image using a combination of edge detection 
and morphological processing, as described schematically in FIG. 8. A Prewitt filter was applied to detect the edges of the bright particles on contrasting dark background. The resulting particle outlines were filled then eroded and dilated using a two-by-two pixel filter to remove spurious pixels. The remaining connected regions of light pixels, each representing a potential particle, were then fit with an ellipse using MATLAB's best-fit functionality (the regionprops routine). Potential particles whose representative ellipses had an eccentricity greater than 0.97 and a length in the range of 4 to $8 \mu \mathrm{m}$ were accepted as valid particle images. The centroid coordinates and orientation of each particle were then calculated from the remaining representative ellipses (regionprops routine outputs).

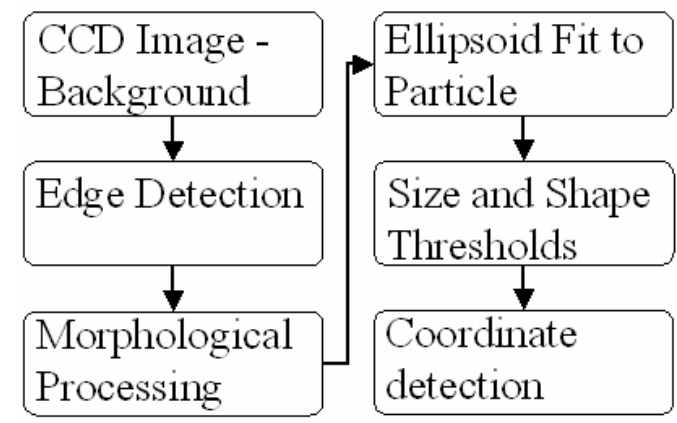

FIG. 8. Image analysis flow chart. First, the background image from each experiment was subtracted from an individual image. Edge detection with a Prewitt filter was utilized to determine particle outlines. These outlines were enhanced and filled using morphological processing including erosion and dilation. The remaining connected regions of light pixels were then fit with an ellipse to determine size and shape for thresholding. Particles whose representative ellipses had an eccentricity greater than 0.97 and a length in the range of 4 to $8 \mu \mathrm{m}$ were accepted as valid particle images. The center coordinates and orientation were determined for these particles from the representative ellipse.

Particle angular and translational displacements between image pairs were calculated for particles which met a "best match" criterion. The matching scheme used a $\chi$-squared test based on the position and orientation of the particle

$$
\chi^{2}=\left(\frac{\left(x_{2}-x_{1}\right)^{2}}{\sigma_{x}^{2}}+\frac{\left(y_{2}-y_{1}-\Delta y_{P I V}\right)^{2}}{\sigma_{y}^{2}}+\frac{\left(\theta_{2}-\theta_{1}\right)^{2}}{\sigma_{\theta}^{2}}\right)
$$

where the subscripts 1 and 2 refer to the image in which the particle was found, $x$ and $y$ are the horizontal and vertical coordinates respectively, theta is the particle angle, and $\sigma_{x}$, $\sigma_{y}$, and $\sigma_{\theta}$ are the variances of the locations and angles of all particles in the second image. A similar approach was used by Takehara et al. [64]. We used micron-resolution particle image velocimetry [65] (using the entire image as the interrogation area) to estimate the uniform average settling drift velocity for the particle field. The vertical shift in the cross-covariance peak between two full images was used to determine the average vertical translation due to gravity. The vertical particle shift resulting from a cross-covariance of the full images of succeeding image pairs is $\Delta y_{P I V}$ in Eq. 43. The $\chi$ - 
squared matching scheme was sufficient for this application as particle-to-particle distances were typically $6 \mu \mathrm{m}$ or larger.

We quantified the accuracy of the PTV code using synthetic images with added Gaussian white noise to mimic images from the experiments. The PTV code located $62 \%$ of particles in the simulated images. The code accurately detects the location of particles with an uncertainty of approximately one half-pixel in the $\mathrm{x}$ or $\mathrm{y}$ direction and an uncertainty in orientation of 0.02 radians.

\section{Diffusivity Measurements}

We investigated the rotational dynamics of Ag particles to measure the rotational diffusivity in the absence of a field. These measurements also provided information regarding contributions of image noise (including the effects of out-of-focus particle regions) to the diffusivity measurements. The particles were imaged at frame rates from 2 to $20 \mathrm{fps}$ while settling through the capillary. The rotational deflections of the particles between images were calculated with the PTV code. These deflection results were combined over all images (and across particles) at a fixed frame rate and the statistical variance in deflection was calculated. FIG. 9 shows a plot of rotational deflection variance versus time between frames $\left(f p s^{-1}\right)$. These data were then fit with linear regression.

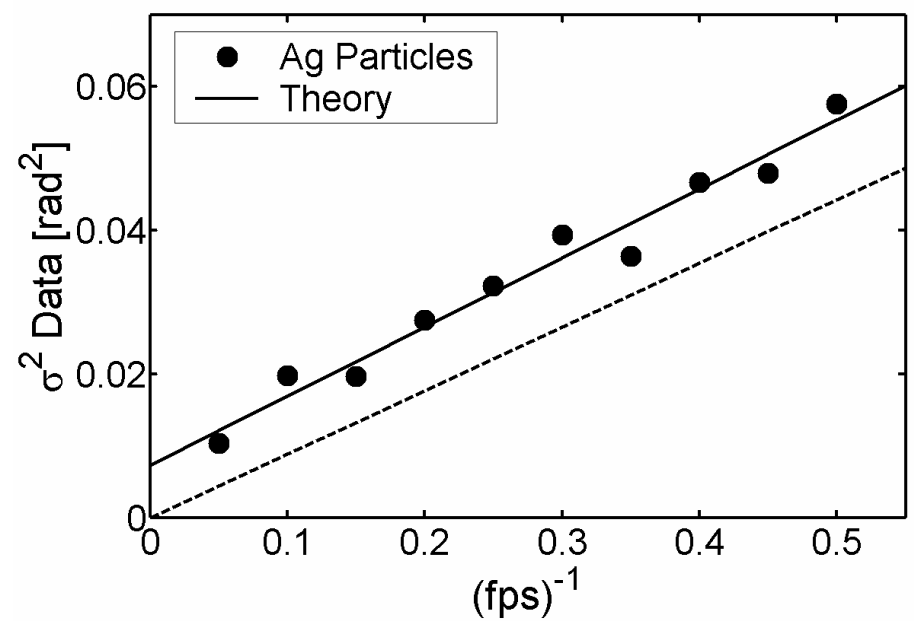

FIG. 9. Variance of angular deflections for Ag particles versus the time between deflection measurements. The slope of the experimental data for the particles is determined using a linear fit. The slope of the data set determines the rotational diffusivity. The solid line is the theoretical curve for diffusivity of these $6 \mu \mathrm{m}$ long by $300 \mathrm{~nm}$ diameter cylindrical particles. The y-offset of the experimental curve at $(\mathrm{fps})^{-1}=0$ is due to image noise.

We model the contributions to particle rotation from image noise and particle diffusion as follows: 


$$
\sigma_{\theta \text { measured }}^{2}=2 D_{\theta} t+\sigma_{\theta \text { noise }}^{2}
$$

where $\sigma_{\theta}^{2}$, is the variance of the deflections, and $t$ is the time between measurements. This additive model is suggested by models used in estimating the contribution of image noise to spherical particle displacements [66]. FIG. 9 shows variance data for the rotation of $\mathrm{Ag}$ particles. Image noise results in a measurable y-intercept for the extrapolated rotational variance data. We can use the regression fit to solve for diffusivity in Eq. 44 and interpret this as data normalized to exclude image noise in the particle rotational displacement statistics.

We compare the measured diffusivity value for the experimental conditions to theoretical values. The experimental diffusivity value for the $\mathrm{Ag}$ particles was $0.048 \mathrm{rad}^{2} / \mathrm{s}$. The theoretical value for a cylindrical particle of this size, $0.044 \mathrm{rad}^{2} / \mathrm{s}$, is determined using the relation given by Yamakawa [67].

$$
D_{\theta}=\frac{3 k T}{\pi \eta L^{3}}[\ln (L / 2 b)+2 \ln 2-11 / 6] .
$$

Our experimental measurement is $9 \%$ greater than the theoretical value for a cylindrical particle and $14 \%$ less than the theoretical diffusivity for a spheroidal particle of equal length and diameter, $0.056 \mathrm{rad}^{2} / \mathrm{s}$, from Eq. 37 .

Image noise also contributes to measurements of particle alignment under applied electric fields. Both rotational diffusion and image noise broaden measured orientation distribution peaks. We incorporate the contribution of image noise to predicted particle distributions by adding the effective diffusivity due to image noise to the theoretical diffusivity predicted by Eq. 37 or 45 . This enables direct comparisons of the model and experimental data. The effective diffusivity due to image noise is defined simply as $D_{\theta, n}=\sigma_{\theta \text { measured }}^{2} /(2 t)$. The data of FIG. 9 yields an image-noise-based diffusivity value of $D_{\theta, n}=0.59 \mathrm{rad}^{2} / \mathrm{s}$, approximately $20 \%$ greater actual rotational diffusivity. This value can perhaps be reduced using larger numerical aperture and larger magnification objectives but only at the expense of reduced fields of view and significantly less particle data per image sequence. The capillary size used in these experiments was selected to minimize particle-wall interaction and necessitated the use of a relatively large working distance objective to image the center plane. The optics optimized the tradeoff between NA, magnification, and working distance.

\section{RESULTS AND DISCUSSION}

The measured orientation distributions of $6 \mu \mathrm{m}$ long by $300 \mathrm{~nm}$ diameter particles were compared versus analytical values at AC field strengths ranging from 10-90 V/cm. The experimental orientation distributions were determined by binning measured particle angles in all images into forty-one, 4.4 degree increments for each field strength. We performed these measurements for both silver and half gold/half silver particles. 

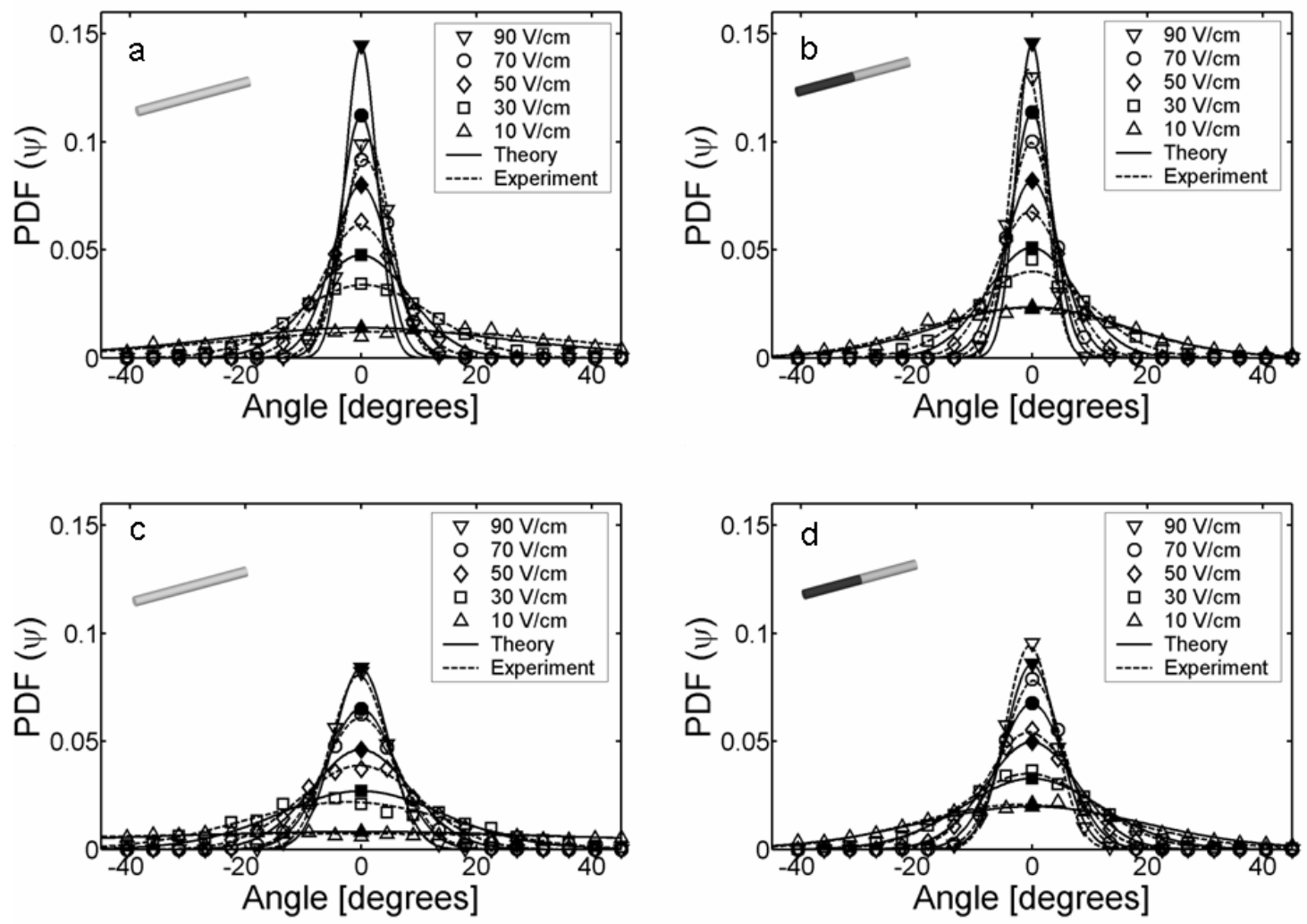

FIG. 10. Comparison of theoretical and experimental orientation distributions at five field strengths for pure silver particles and half gold / half silver particles with thin EDL $\left(\lambda_{D} / b=0.17\right)$ and intermediate EDL $\left(\lambda_{D} / b=0.91\right)$. The experimental data are shown using dashed lines and open symbols at selected angles. The solid lines with solid symbols are the analytical solutions to the Fokker-Planck equation for the distribution of particle angles. The full range of the plot (-90 to 90 degrees) is not shown. a) Pure silver particles with thin EDL $\left(\lambda_{D} / b=0.17\right)$. b) Half gold / half silver particles with thin EDL $\left(\lambda_{D} / b=0.17\right)$. c) Pure silver particles with intermediate EDL $\left(\lambda_{D} / b=0.91\right)$. d) Half gold / half silver particles with intermediate $\operatorname{EDL}\left(\lambda_{D} / b=0.91\right)$.

To achieve a relatively thin EDL the particles were suspended in $0.14 \mathrm{mM} \mathrm{KCl}$ solution. This concentration was selected due to significant particle agglomeration in higher conductivity solutions. The double layer thickness for this solution was approximately $26 \mathrm{~nm}$, resulting in a $\lambda_{D} / b$ ratio equal to 0.17 . Values for $\left(\lambda_{D} / b\right) \exp \left(z e\left(\zeta_{o}+E_{\infty} c\right) / 2 k T\right)$, regarding the applicability of Eq. 1 for the translation and Eqs. $19 \mathrm{~b}$ and $20 \mathrm{~b}$ for the rotation, ranged from 0.3-0.5. This range is based on the sum of a native zeta potential of approximately $30 \mathrm{mV}$ and an induced zeta potential magnitude, $\zeta \cong E_{\infty} c$, ranging from 3 to $27 \mathrm{mV}$. To achieve a relatively thick EDL the particles were suspended in low conductivity $(2 \mu \mathrm{S} / \mathrm{cm})$ deionized water. This ion concentration was the minimum achievable due to carbon dioxide contamination from the atmosphere. The 
double layer thickness for particles in the deionized water was approximately $135 \mathrm{~nm}$ for a $\lambda_{D} / b$ ratio equal to 0.91 and $\lambda_{D} / c$ ratio equal to 0.05 . In this estimate of the double layer thickness, we assumed the ions in the water were solely from carbon dioxide contamination creating carbonic acid in the water [68]. The minimum and maximum $\lambda_{D} / b$ values were also limited by the available particle size. A compromise was therefore made to use representative $\lambda_{D} / b$ values to model the thick and thin double layer limits.
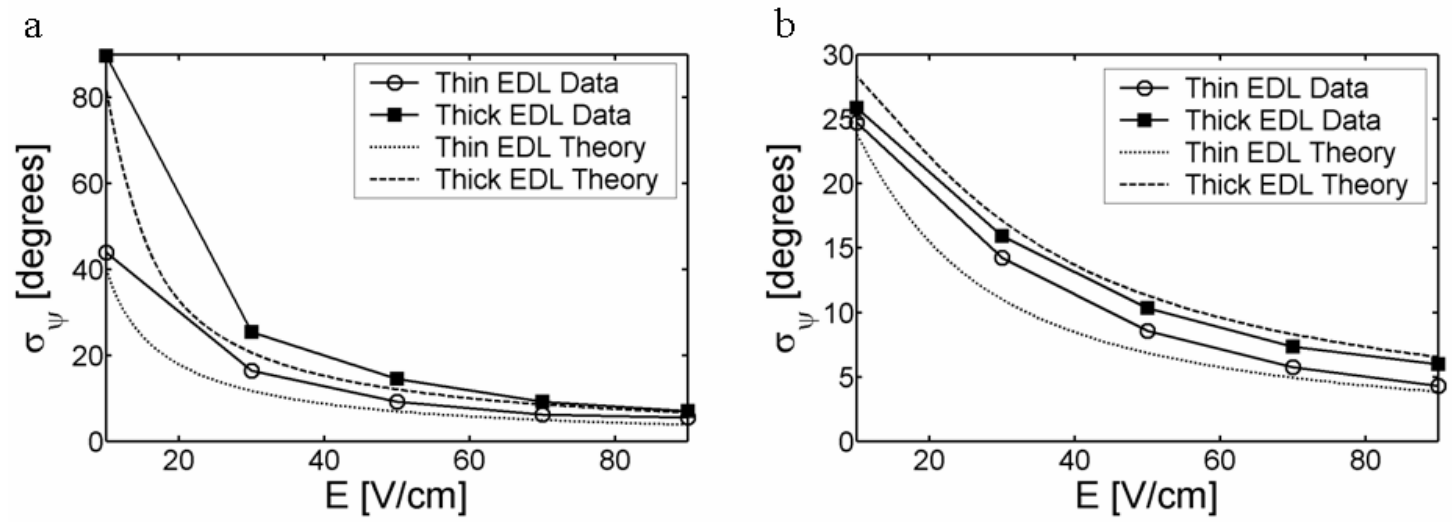

FIG. 11. Standard deviation of particle angles for $\mathrm{Ag}$ (a) and $\mathrm{Ag} / \mathrm{Au}$ (b) particles in high and low conductivity solutions with different applied field strengths. Experimental values are calculated directly from the orientation data of all particles at a given field strength. The theoretical curves are determined from the predicted orientation distributions with the $\mathrm{AgAu}$ curves including predicted alignment due to gravitational torque.

The results of the pure silver particle experiments in $\mathrm{KCl}$ solution are shown in FIG. 10a and for half gold / half silver in FIG. 10b. As the field strength increases, the distribution curves for both particle types narrow. The alignment into the field is enhanced by the increase in induced zeta potential and by the stronger field acting on this polarized charge. For $\mathrm{Ag} / \mathrm{Au}$ particles, the particles are even more aligned than the homogenous Ag particles at the same field strength. This increased alignment is due to the additional rotation generated by gravity acting on the non-uniform density of the particles.

The orientation distribution for pure silver particles in DI water is shown versus theoretical values in FIG. 10c and for half gold/half silver in FIG. 10d. For the particles in DI water, the alignment was enhanced by increased field strength, similar to the results for the particles in $\mathrm{KCl}$ solution. The alignment of the half $\mathrm{Au} /$ half $\mathrm{Ag}$ particles is greater than that of the pure $\mathrm{Ag}$ particles due to the additional torque from gravity.

FIG. 11 shows plots of the standard deviation of particle angles versus electric field for the homogenous and striped particles in high and low conductivity solutions. Experimental values are calculated directly from the orientation data of all particles at a given field strength. The theoretical curves are determined from Eqs. 5b, 27b, 32b, and 40, and relevant parameters as described above. 
As expected, the gravitational torque due to material mismatch of the half gold / half silver particles increases the magnitude of the rotational velocity compared to pure silver particles. This is true for both the high and low conductivity solutions. Another interesting observation is the increased $P e_{p o l}$ for the particles with thin double layers versus the same particle type with a thick EDL. The increase in the $P e_{p o l}$ value for thin EDL demonstrates the magnitude of ICEP rotation on the particle.

Analytical values for the orientation distributions, $\psi(\theta)$, were predicted using Equation 40 with Peclet number values, $P e_{p o l}$ and $P e_{p e r m}$. These $P e$ values were determined based on the theory for $\omega_{\text {pol }}$ and $\omega_{\text {grav }}$ and independent estimates of the relevant parameters. These independent estimates are the particle dimensions (from the specification of the manufacturer), the electrolyte ion density (from our buffer preparation), and the rotational diffusivity. We used the experimentally measured particle diffusivity values in the analytical prediction of $P e$ to account for image noise. The justification for this modification is explained in detail in Section III-C. The value for the rotational velocity in $P e_{p o l}$ was predicted from the time-average of Eq. $27 \mathrm{~b}$ for the thin EDL model. For the thick EDL model, the rotational velocity in $P e_{p o l}$ was predicted from the time-average of Eq. $5 \mathrm{~b}$ using the DEP torque value from Eq. $7 \mathrm{~b}$. The rotational velocity in $P e_{\text {grav }}$ was predicted from Eq. $32 \mathrm{~b}$ with specific gravity of gold, $s g_{A u}=19.3$, and silver, $s g_{A g}=10.5$ [69].

The analytical results for the thin EDL particles at field strengths ranging from $10-90 \mathrm{~V} / \mathrm{cm}$ are shown using solid lines with closed symbols in FIG. 10a-b. The model provides an upper bound for the alignment of the particles as $\lambda_{D} / b \rightarrow 0$ and therefore consistently over predicts alignment of the particles for the given experimental conditions, $\lambda_{D} / b=0.17$. The model does however predict the increase in particle alignment with increased field strength due to polarization. This is demonstrated by the narrowing of the distribution for the Ag particles in FIG. 10a. The model also captures the combined effects of polarization and gravity at low field strength $(10 \mathrm{~V} / \mathrm{cm})$ as demonstrated in FIG. 10b for the half gold / half silver particles.

The solid lines with closed symbols in FIG. 10c and FIG. 10d show the analytical results for $\psi(\theta)$. For the thick EDL case, the experimental and theoretical curves show good agreement for both $\mathrm{Ag}$ and $\mathrm{Ag} / \mathrm{Au}$ particles. The agreement is best for $\mathrm{Ag} / \mathrm{Au}$ particles at low field strengths $(10-30 \mathrm{~V} / \mathrm{cm})$. The predicted increase in particle alignment due to increased polarization is demonstrated by the narrowing of the distribution for the Ag particles in FIG. 10c. The additional increase in alignment due to non-uniform density is also captured in the model. This result is verified in FIG. 10d for the half gold / half silver particles.

FIG. 11a and FIG. 11b compare the predicted and experimental dependence of the standard deviation in particle angles on electric field strength for $\mathrm{Ag}$ and $\mathrm{Ag} / \mathrm{Au}$ particles. For $\mathrm{Ag}$ and $\mathrm{Ag} / \mathrm{Au}$ particles with thick EDL, the experimental results are predicted within $10 \%$ over the range of applied fields. For thin EDL, the theoretical $\sigma_{\psi}$ curve provides a lower bound for predicting the experimental values. The predictions for the $\mathrm{Ag} / \mathrm{Au}$ particles include the rotation of the particles due to gravity.

\section{CONCLUSION}

We have presented analytical results for the rotation of homogeneous and striped rod-like metal particles in an applied field for the limiting cases of thin $\left(\lambda_{D}<<b\right)$ and 
thick $\left(\lambda_{D}>>c\right)$ electric double layers. The two models include rotational velocity components from (uniform or non-uniform) native surface charge along the particle length and the non-uniform induced surface charge due to the conducting nature of the particle. The distribution of particle orientations in a dilute suspension is determined from the balance of electric field driven rotation and rotational diffusion. This distribution is especially important for solutions with thick EDL, in which electrophoretic translation of rod-like particles is orientation dependent.

The experimental results were presented for solid $\mathrm{Ag}$ and for half $\mathrm{Ag} / \mathrm{half} \mathrm{Au}$ particles in relatively thin and relatively thick EDL conditions are in good agreement with the analytical models for the thick EDL case. For the thin EDL case, the analytical models over predict alignment. This is likely due to the experimental $\lambda_{D} / b$ ratio equal of 0.17 although the model is for $\lambda_{D} / b \rightarrow 0$. For particles with striping patterns that are not symmetric about particle length, the net effect of the stripes is to impart a torque on the particle due to gravity. This dipole causes a rotation of the particle that enhances alignment. This enhancement is demonstrated by comparison of results for solid Ag and half $\mathrm{Ag} / \mathrm{half} \mathrm{Au}$ particles. The electric torque on the particle due to induced polarization is also shown to be greater for particles with thin versus thick electric double layers. This result is analogous to the comparison of the translational electrophoretic velocity of spheres in the thin and thick EDL limits.

\section{ACKNOWLEDGEMENTS}

This work was performed under the auspices of the U.S. Department of Energy by University of California, Lawrence Livermore National Laboratory under Contract W-7405-Eng-48. 


\section{REFERENCES}

[1] C.D. Keating and M.J. Natan, Adv. Mater. 15, 451 (2003).

[2] I.D. Walton, S.M. Norton, A. Balasingham, L. He, D.F. Oviso, D. Gupta, P.A. Raju, M.J. Natan, and R.G. Freeman, Anal. Chem. 74, 2240 (2002).

[3] S.R. Nicewarner-Pena, R.G. Freeman, B.D. Reiss, L. He, D.J. Pena, I.D. Walton, R. Cromer, C.D. Keating, and M.J. Natan, Science 294, 137 (2001).

[4] J. Hahm and C.M. Lieber, Nanoletters 4, 51 (2004).

[5] L.A. Bauer, N.S. Birenbaum, and G.J. Meyer, J. Mater. Chem. 14, 517 (2004).

[6] J.F. Klemic, E. Stern, and M.A. Reed, Nat. Biotechnol. 19, 924 (2001).

[7] Y. Huang, X. Duan, Y. Cui, L.J. Lauhon, K.-H. Kim, and C.M. Lieber, Science 294, 1313 (2001).

[8] K.D. Hermanson, S.O. Lumsdon, J.P. Williams, E.W. Kaler, and O.D. Velev, Science 294, 1082 (2001).

[9] D.H. Reich, M. Tanase, A. Hultgren, L.A. Bauer, C.S. Chen, and G.J. Meyer, J. Appl. Phys. 93, 7275 (2003).

[10] S. Evoy, et al., Microelectron. Eng. 75, 31 (2004).

[11] P.A. Smith, C.D. Nordquist, T.N. Jackson, T.S. Mayer, B.R. Martin, J. Mbindyo, and T.E. Mallouk, Applied Physics Letters 77, 1399 (2000).

[12] S.M. Davison, T.S. Mayer, and K.V. Sharp, in ASME,(Anaheim, CA, 2004)

[13] B.M.I. Van der Zande, J.K.G. Dhont, M.R. Böhmer, and A.P. Philipse, Langmuir 16, 459 (1999).

[14] J. Lyklema, Solid-Liquid Interfaces, Fundamentals of Interface and Colloid Science (Academic Press, San Diego, 1995), Vol. 2.

[15] J.L. Anderson, J. Colloid Interf. Sci. 105, 45 (1985).

[16] A. Sellier, Q. J. Mech. Appl. Math. 55, 561 (2002).

[17] R.J. Hunter, Zeta potential in colloidal science (Academic Press, San Diego, 1981).

[18] F.A. Morrison, J. Colloid Interf. Sci. 34, 210 (1970).

[19] M. Teubner, J. Chem. Phys. 76, 5564 (1982).

[20] E. Huckel, Physik. Zeitsch. 25, 204 (1924).

[21] L.B. Harris, J. Colloid Interf. Sci. 34, 322 (1970).

[22] D.C. Henry, P. Roy. Soc. Lond. A Mat. 133, 106 (1931).

[23] H. Ohshima, J. Colloid Interf. Sci. 180, 299 (1996).

[24] B.J. Yoon and S. Kim, J. Colloid Interf. Sci. 128, 275 (1989).

[25] D. Stigter, J. Phys. Chem. 82, 1417 (1978).

[26] D. Stigter, J. Phys. Chem. 82, 1424 (1978).

[27] N.A. Mishchuk and S.S. Dukhin, Colloid J.-USSR 50, 952 (1988).

[28] R.W. O'Brien and D.N. Ward, J. Colloid Interf. Sci. 121, 402 (1988).

[29] C.C. Ho, R.H. Ottewill, and L. Yu, Langmuir 13, 1925 (1997).

[30] S.S. Dukhin, Adv. Colloid Interfac. 44, 1 (1993).

[31] S.P. Stoilov and S.S. Dukhin, Colloid J.-USSR 32, 631 (1970).

[32] V.R. Estrela-Lopis, V.N. Shilov, S.S. Dukhin, and S.P. Stoilov, Colloid J.-USSR 35, 578 (1973).

[33] V.N. Shilov, Y.Y. Rozen, and S.S. Dukhin, Colloid J.-USSR 36, 1133 (1974).

[34] V.V. Malyarenko, F.D. Ovcharenko, and V.P. Estrella-Lopez, Dokl. Akad. Nauk. $\mathrm{SSSR}+$ 215, 321 (1974). 
[35] M. Fixman, J. Chem. Phys. 72, 5177 (1980).

[36] M. Fixman, Macromolecules 13, 711 (1980).

[37] S.S. Dukhin and V.N. Shilov, Adv. Colloid Interfac. 13, 153 (1980).

[38] M. Mandel and T. Odijk, Annu. Rev. Phys. Chem. 35, 75 (1984).

[39] I.N. Simonov and S.S. Dukhin, Colloid J.-USSR 35, 173 (1973).

[40] T.M. Squires and M.Z. Bazant, J. Fluid Mech. 509, 217 (2004).

[41] M. Fixman and S. Jagannathan, J. Chem. Phys. 75, 4048 (1981).

[42] M.Z. Bazant and T.M. Squires, Phys. Rev. Lett. 92, 066101/1 (2004).

[43] T.M. Squires and M.Z. Bazant, J. Fluid Mech., in press (2006).

[44] E. Yariv, Phys. Fluids 17, 051702 (2005).

[45] D. Saintillan, E. Darve, and E. Shaqfeh, J. Fluid Mech. 563, 223 (2006).

[46] A. de Keizer, W.P.J.T. van der Drift, and J.T.G. Overbeek, Biophys. Chem. 3, 107 (1975).

[47] S.P. Han and S.-M. Yang, J. Colloid Interf. Sci. 177, 132 (1996).

[48] M. Abramowitz and I.A. Stegun, Hanbook for Mathematical Functions with Formulas, Graphs, and Mathematical Tables (Dover, New York, 1964).

[49] N.J. Rivette and J.C. Baygents, Chem. Eng. Sci. 51, 5205 (1996).

[50] J. T.B., IEEE Engineering in Medicine and Biology Magazine 22, 33 (2003).

[51] J.A. Stratton, Electromagnetic Theory (McGraw-Hill, New York, 1941).

[52] L.D. Landau and E.M. Lifshitz, Electrodynamics of Continuous Media (Permagon Press, Oxford, 1960), Vol. 8.

[53] J.H. Jeans, The Mathematical Theory of Electricity and Magnetism (The University Press, Cambridge, 1925).

[54] H. Lamb, Hydrodynamics (Cambridge Univ. Press, Cambridge, 1932).

[55] J. Happel and H. Brenner, Low Reynolds Number Hydrodynamics (Martinus Nijhoff Publishers, Boston, 1983).

[56] M. Doi, The Theory of Polymer Dynamics (Oxford University Press, Oxford, 1988).

[57] D. Stigter, J. Phys. Chem. 86, 3553 (1982).

[58] T.G.M. van de Ven, Chem. Eng. Sci. 56, 2947 (2001).

[59] T.M. Squires and M.Z. Bazant, J. Fluid Mech. (Submitted 20 July 2005).

[60] M.C. Fair and J.L. Anderson, J. Colloid Interf. Sci. 127, 388 (1989).

[61] Y.K. Chen and C.P. Yu, Aerosol Sci. Tech. 16, 255 (1992).

[62] Y.P. Kalmykov, J. Mol. Liq. 69, 117 (1996).

[63] P.S. Grassia, E.J. Hinch, and L.C. Nitsche, J. Fluid Mech. 282, 373 (1995).

[64] K. Takehara, R.J. Adrian, G.T. Etoh, and K.T. Christensen, Exp. Fluids S34 (2000).

[65] J.G. Santiago, S.T.Wereley, C.D. Meinhart, D.J. Beebe, and R.J. Adrian, Exp. Fluids 25, 316 (1998).

[66] M. Raffel, C. Willert, and J. Kompenhans, Particle Image Velocimetry: A Practical Guide. (Springer-Verlag, Berlin, 1998).

[67] H. Yamakawa, Macromolecules 8, 339 (1975).

[68] S.L. Zeng, C.H. Chen, J.C. Mikkelsen, and J.G. Santiago, Sensor Actuat. BChem. 79, 107 (2001). 
[69] Physical Constants of Organic Compounds, in CRC Handbook of Chemistry and Physics, Internet Version 2007, (87th Edition), D.R. Lide, Editor, Taylor and Francis: Boca Raton, FL. 